\title{
Reappraisal of the Kelvin-Helmholtz problem. Part 1. Hamiltonian structure
}

\author{
By T. BROOKE BENJAMIN ${ }^{1} \dagger$ AND THOMAS J. BRIDGES \\ ${ }^{1}$ Mathematical Institute, Oxford University, 24-29 St. Giles, Oxford OX1 3LB, UK \\ ${ }^{2}$ Department of Mathematical and Computing Sciences, University of Surrey, Guildford, \\ Surrey GU2 5XH, UK
}

(Received 15 November 1990 and in revised form 12 September 1996)

This paper and Part 2 report various new insights into the classic Kelvin-Helmholtz problem which models the instability of a plane vortex sheet and the complicated motions arising therefrom. The full nonlinear version of the hydrodynamic problem is treated, with allowance for gravity and surface tension, and the account deals in precise fashion with several inherently peculiar properties of the mathematical model. The main achievement of the paper, presented in $\S 3$, is to demonstrate that the problem admits a canonical Hamiltonian formulation, which represents a novel variational definition of a functional representing perturbations in kinetic energy. The Hamiltonian structure thus revealed is then used to account systematically for relations between symmetries and conservation laws, and none of those examined appears to have been noticed before. In $\S 4$, a generalized, non-canonical Hamiltonian structure is shown to apply when the vortex sheet becomes folded, so requiring a parametric representation, as is well known to occur in the later stages of evolution from Kelvin-Helmholtz instability. Further invariant properties are demonstrated in this context. Finally, $\S 5$, the linearized version of the problem - reviewed briefly in $\$ 2.1$ - is reappraised in the light of Hamiltonian structure, and it is shown how Kelvin-Helmholtz instability can be interpreted as the coincidence of wave modes characterized respectively by positive and negative values of the Hamiltonian functional representing perturbations in total energy.

\section{Introduction}

Named after Kelvin and Helmholtz who developed it originally, the linearized theory describing the instability of a plane vortex sheet is a classic of hydrodynamics. Accounts of it are included in many standard texts (e.g. Lamb 1932, §232). Being the subject of many further theoretical developments to be reappraised here (e.g. Drazin 1970), the nonlinear evolution of perfect-fluid motions started from small perturbations of a vortex sheet is also usually known by the term Kelvin-Helmholtz instability; and the general form of behaviour exhibited in later stages of evolution, in which the sheet rolls up into billows, is well known from numerical solutions of the hydrodynamic problem. In the original model the unperturbed vortex sheet is horizontal, separating an infinite perfect fluid below from another perfect fluid of smaller density above, and so gravity has some stabilizing influence. The model is nevertheless pathological in that, however small the discontinuity in tangential velocity 
across it, the interface is unstable and the growth rate of infinitesimal disturbances increases without limit as their wavelength decreases. Allowance for surface tension obviates these unsatisfactory features, and the Kelvin-Helmholtz model with this straightforward modification will be the subject of the extensive reassessment that follows here and in Part 2 (Benjamin \& Bridges 1997).

At first sight the evolutionary problem posed by this model appears peculiarly complicated, lacking standard attributes of nonlinear dynamical systems. It appears to be much more complicated than the problem of water waves, to which of course the present problem reduces, losing its distinctive aspects if the upper fluid is removed. The perturbed motions in the lower and upper fluids are both irrotational, but each of their respective velocity potentials has an intricate, different relation to the configuration and normal velocity of the interface; in general neither the potentials themselves nor their normal derivatives are continuous across the interface. Moreover, the dynamical condition relating pressures in the fluids on either side of the interface to the normal-stress discontinuity caused by surface tension is formidably complex; and thus the underlying simple structure that will be revealed in what follows is by no means obvious.

It will be shown in $\S 3$, for the first time as far as we know, that the full nonlinear problem admits a canonical Hamiltonian formulation. This achievement depends on recognizing a suitable pair of dependent variables, which alone determine every other property of the motion, and on defining a suitable Hamiltonian functional which represents a bounded and conserved perturbation of energy. (The total kinetic energy of the Kelvin-Helmholtz model is of course infinite in any frame of reference.) A crucial step is the use of a variational principle to define the kinetic-energy perturbation as a functional of the dependent variables, which device is believed to be novel. The device is essential to our theory, being the cornerstone of the discovered Hamiltonian structure, and it can be applied as a refinement of other Hamiltonian formulations for continuous (i.e. infinite-dimensional) dynamical systems - notably that for the water-wave problem.

Because the water-wave problem is included as a degenerate case of the KelvinHelmholtz problem, the present formulation may be regarded as a generalization of the simpler case and advantages comparable at least with those provided in this way for water-wave theory may be sought. The Hamiltonian representation for irrotational water waves was noticed first by Zakharov (1968), and it has been variously used by Benjamin (1972, 1974), Miles (1977), Benjamin \& Olver (1982) and others. The addition of vorticity requires a Lie-Poisson formulation and, for inviscid water waves, this structure has been constructed by Lewis et al. (1986).

An important consequence of Hamiltonian structure is a framework for enumerating conservation laws. For example, Benjamin \& Olver used the Hamiltonian structure of the irrotational water-wave problem to account systematically for relations between its symmetries and all the conservation laws for water waves. On a similar basis, for the Kelvin-Helmholtz problem, a number of interesting conservation laws related to symmetries will be demonstrated in $\S 3.3$ and $\$ 4.3$; but we do not claim the list of such properties to be complete. Two functionals constituting the components of horizontal impulse are of special interest, being confirmed as invariants of any free motion. They are identified according to a generalized definition of impulse with reference to Hamiltonian structure and the horizontal translation group (cf. Benjamin 1984, §1.3), and they are perhaps surprising because a rudimentary definition of hydrodynamic impulse (e.g. see Lamb 1932, §152) fails to have meaning when applied to the present model. 


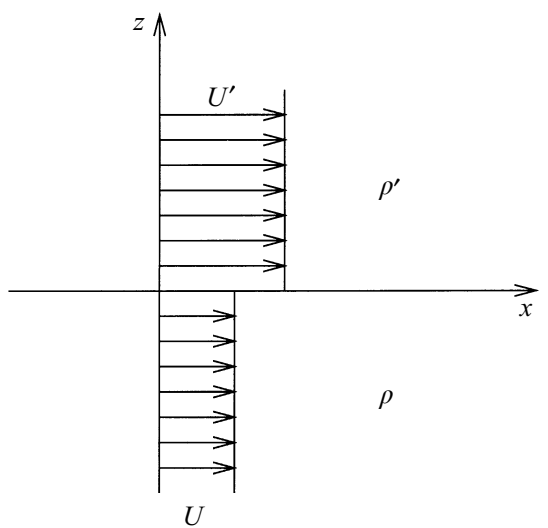

Figure 1. Illustration of the Kelvin-Helmholtz model's undisturbed state.

The canonical Hamiltonian formulation established in $\$ 3$ ceases to hold when, as is well known to happen eventually as an outcome of Kelvin-Helmholtz instability, the interface becomes folded so that its height is no longer a single-value function of horizontal position. An extension of the new theory to this state of evolution will be presented in $\S 4$, where a non-canonical but equally useful Hamiltonian formulation will be shown to hold for parametric representations of the evolving interface. This formulation generalizes one that Benjamin \& Olver (1982, Appendix 1) showed to cover parametric representations of the free surface in the water-wave problem, and it too provides a clear, indeed particularly conspicuous, view of the relations between symmetries and conservation laws. The principal conservation laws demonstrated may deserve attention as checks on numerical investigations into the later stages of evolution from Kelvin-Helmholtz instability.

We accordingly claim that this classic problem still offers much scope for new insights. Other novel aspects, several of them surprising, will be presented in Part 2.

\section{The hydrodynamic problem}

The model demonstrating Kelvin-Helmholtz instability is illustrated in figure 1. In the model's undisturbed state, the horizontal plane $z=0$ coincides with the interface between perfect liquids of density $\rho$ below and density $\rho^{\prime}<\rho$ above, which have velocities $U$ and $U^{\prime}$ respectively in the $x$-direction. The disturbed interface $S$ is described by

$$
z=\eta(x, y, t)
$$

where $\eta$ is supposed to be a single-valued, continuously differentiable function of the horizontal coordinates $(x, y)$. (In $\S 4$ it will be shown how the theoretical problem is modified when $S$ is folded and a parametric description of $S$ becomes necessary.) Perturbations of the flows below and above $S$ are assumed to be irrotational, having velocity potentials $\phi(x, y, z, t)$ and $\phi^{\prime}(x, y, z, t)$ which are harmonic functions of $(x, y, z)$ in the respective regions $z<\eta$ and $z>\eta$. The liquids are taken to be unbounded below and above $S$, so that we require $|\nabla \phi| \rightarrow 0$ as $z \rightarrow-\infty$ and $\left|\nabla \phi^{\prime}\right| \rightarrow 0$ as $z \rightarrow \infty$.

Let $\Phi_{(n)}$ and $\Phi_{(n)}^{\prime}$ denote the normal derivatives of $\phi$ and $\phi^{\prime}$ at $S$, where the normal on $S$ points into the upper fluid. Then the kinematic conditions relating the motion 
of $S$ to velocities in the two contiguous liquids can be written

$$
\eta_{t}+U \eta_{x}=R \Phi_{(n)}, \quad \eta_{t}+U^{\prime} \eta_{x}=R \Phi_{(n)}^{\prime},
$$

with $R=\left(1+\eta_{x}^{2}+\eta_{y}^{2}\right)^{1 / 2}$ (cf. Drazin 1970, equation (5)). The dynamical condition at the interface is found upon using the Bernoulli integral, for the irrotational motions on each side, to express the pressure difference $p-p^{\prime}$ across $S$. Allowance is made that the stagnation pressure (Bernoulli constant) is $\frac{1}{2} \rho U^{2}$ below the interface, $\frac{1}{2} \rho^{\prime} U^{\prime 2}$ above, and that $p-p^{\prime}=-2 \sigma H$, where $\sigma$ is the coefficient of surface tension and

$$
2 H=\left(\eta_{x} / R\right)_{x}+\left(\eta_{y} / R\right)_{y}
$$

is twice the (upward) mean curvature of $S$. The outcome is

$$
\rho\left(\Phi_{(t)}+U \Phi_{(x)}+\frac{1}{2} q^{2}\right)-\rho^{\prime}\left(\Phi_{(t)}^{\prime}+U^{\prime} \Phi_{(x)}^{\prime}+\frac{1}{2} q^{\prime 2}\right)=-g\left(\rho-\rho^{\prime}\right) \eta+2 \sigma H,
$$

in which $\Phi_{(t)}=\phi_{t}(x, y, \eta, t), \Phi_{(x)}=\phi_{x}(x, y, \eta, t), q=|\nabla \phi|$ at $z=\eta$, and correspondingly for derivatives of $\phi^{\prime}$ (cf. Drazin 1970, equation (6)).

The nonlinear conditions (2.1) and (2.2) completely determine the evolution of the surface $S$ until $R \rightarrow \infty$ somewhere on it and thereafter the surface becomes folded. The evolutionary problem will be shown in $\S 3$ to have a canonical Hamiltonian representation in terms of the dependent variables $\eta$ and $\zeta=\rho \Phi-\rho^{\prime} \Phi^{\prime}$, where $\Phi=\phi(x, y, \eta, t)$ and $\Phi^{\prime}=\phi^{\prime}(x, y, \eta, t)$.

\subsection{Linearized theory}

The results given upon linearisation of (2.1) and (2.2) are classic (cf. Lamb 1932; Chandrasekhar 1961, §101), but they need to be recalled for reference later. The linearized versions of (2.1) and (2.2) are

$$
\begin{gathered}
\eta_{t}+U \eta_{x}=\left.\phi_{z}\right|_{z=0}, \quad \eta_{t}+U^{\prime} \eta_{x}=\left.\phi_{z}^{\prime}\right|_{z=0}, \\
\left.\rho\left[\phi_{t}+U \phi_{x}\right]\right|_{z=0}-\left.\rho^{\prime}\left[\phi_{t}^{\prime}+U^{\prime} \phi_{x}^{\prime}\right]\right|_{z=0}=-g\left(\rho-\rho^{\prime}\right) \eta+\sigma\left(\eta_{x x}+\eta_{y y}\right) .
\end{gathered}
$$

The representative solution of the linearized problem has the form

$$
\left(\begin{array}{c}
\eta(x, y, t) \\
\phi(x, y, z, t) \\
\phi^{\prime}(x, y, z, t)
\end{array}\right)=\operatorname{Re}\left[\mathrm{e}^{\mathrm{i}(\alpha x+\beta y)}\left(\begin{array}{c}
A(t) \\
B(t) \mathrm{e}^{k z} \\
B^{\prime}(t) \mathrm{e}^{-k z}
\end{array}\right)\right],
$$

where the wavenumbers $\alpha$ and $\beta$ are real and $k=+\left(\alpha^{2}+\beta^{2}\right)^{1 / 2}$. The kinematic conditions (2.3) require that $B=(\dot{A}+\mathrm{i} \alpha U A) / k, B^{\prime}=-\left(\dot{A}+\mathrm{i} \alpha U^{\prime} A\right) / k$; and, if $A(t)=A(0) \mathrm{e}^{\mathrm{st}},(2.4)$ then gives

$$
\rho(s+\mathrm{i} \alpha U)^{2}+\rho^{\prime}\left(s+\mathrm{i} \alpha U^{\prime}\right)^{2}+g\left(\rho-\rho^{\prime}\right) k+\sigma k^{3}=0,
$$

whence

$$
s=-\frac{\mathrm{i} \alpha\left(\rho U+\rho^{\prime} U^{\prime}\right)}{\rho+\rho^{\prime}} \pm\left\{\frac{\alpha^{2} \rho \rho^{\prime}\left(U-U^{\prime}\right)^{2}}{\left(\rho+\rho^{\prime}\right)^{2}}-\frac{g\left(\rho-\rho^{\prime}\right) k+\sigma k^{3}}{\rho+\rho^{\prime}}\right\}^{1 / 2} .
$$

The first, always purely imaginary term on the right-hand side of (2.5) represents convection of any wave at velocity $\left(\rho U+\rho^{\prime} U^{\prime}\right) /\left(\rho+\rho^{\prime}\right)$ in the $x$-direction. Instability is indicated by the second term, with alternative sign, being real. Note that, for any unstable wave with given $k$, its growth rate $\operatorname{Re}(s)>0$ is greatest when $\beta=0$ (i.e. $k=\alpha)$ and the wave is directed parallel to the basic flow. As $\left(U-U^{\prime}\right)^{2}$ is increased, the first wave to become unstable has $\alpha^{2}=g\left(\rho-\rho^{\prime}\right) / \sigma$, and correspondingly the 
condition for instability of the basic flow is that

$$
\left(U-U^{\prime}\right)^{2}>2\left(\frac{\rho+\rho^{\prime}}{\rho \rho^{\prime}}\right)\left[g\left(\rho-\rho^{\prime}\right) \sigma\right]^{1 / 2} .
$$

Further commentary on the classic formula (2.5) will be included in $\S 5$ and in Part 2.

\section{Hamiltonian formulation}

First, in order to define integral properties of the disturbed motion, the relevant range of $(x, y)$ is denoted by $\Omega$, which may have any one of the following meanings. For the case of localized three-dimensional motions evanescent as $\left(x^{2}+y^{2}\right)^{1 / 2} \rightarrow \infty$, it is understood that $\Omega=\mathbb{R} \times \mathbb{R}$. For the case of three-dimensional motions periodic in $x$ and $y$, it is understood that $\Omega=($ period in $x) \times($ period in $y)$. For two-dimensional motions independent of $y$, a unit span in the $y$-direction is implied and then either $\Omega=\mathbb{R}$ or $\Omega=($ period in $x)$.

The disjoint domains in which respectively $\phi$ and $\phi^{\prime}$ are harmonic functions of $(x, y, z)$ are

$$
\mathscr{D}=\Omega \times(-\infty, \eta) \quad \text { and } \quad \mathscr{D}^{\prime}=\Omega \times(\eta, \infty) .
$$

The interface $z=\eta(x, y, t),(x, y) \in \Omega$, between these domains varies with time $t$; but for our immediate purpose the $t$-dependence of $\eta, \mathscr{D}$ and $\mathscr{D}^{\prime}$ can be left implicit.

\subsection{Properties of the kinetic-energy functional}

The kinetic energy of the motion is evidently unbounded unless $U=U^{\prime}=0$. A scalar quantity $K$ can nevertheless be defined representing the kinetic-energy perturbation that occurs when the quiescent state of the model system is disturbed. For reasons to be explained presently, it is expressed by

$K=-\int_{\Omega}\left(\rho U \Phi-\rho^{\prime} U^{\prime} \Phi^{\prime}\right) \eta_{x} \mathrm{~d} x \mathrm{~d} y+\int_{\mathscr{D}} \frac{1}{2} \rho|\nabla \phi|^{2} \mathrm{~d} x \mathrm{~d} y \mathrm{~d} z+\int_{\mathscr{D}^{\prime}} \frac{1}{2} \rho^{\prime}\left|\nabla \phi^{\prime}\right|^{2} \mathrm{~d} x \mathrm{~d} y \mathrm{~d} z$

The appropriateness of this definition is borne out by the analysis that follows. Upon taking the total-energy functional as $E=K+V$, where $V$ represents potential energy, it is found that $E$ has the role of the Hamiltonian in a canonical representation of the governing equations (2.1) and (2.2); and accordingly $E$ is confirmed to be an invariant of any free motion (provided $g$ is independent of $t$ ). In the case that $\phi$ and $\phi^{\prime}$ are periodic in $x$ and $y$, moreover, the identification of (3.2) with the perturbation of kinetic energy is immediate in physical terms. Writing

$$
K=\int_{\mathscr{D}} \rho\left(\frac{1}{2}|\nabla \phi|^{2}+U \phi_{x}\right) \mathrm{d} x \mathrm{~d} y \mathrm{~d} z+\int_{\mathscr{D}^{\prime}} \rho^{\prime}\left(\frac{1}{2}\left|\nabla \phi^{\prime}\right|^{2}+U^{\prime} \phi_{x}^{\prime}\right) \mathrm{d} x \mathrm{~d} y \mathrm{~d} z,
$$

we may note in this case that by Green's theorem

$$
\begin{aligned}
\int_{\mathscr{D}} \phi_{x} \mathrm{~d} x \mathrm{~d} y \mathrm{~d} z & =\int_{\mathscr{D}} \nabla \phi \cdot \nabla x \mathrm{~d} x \mathrm{~d} y \mathrm{~d} z=\int_{S} \Phi \frac{\partial x}{\partial n} \mathrm{~d} s \\
& =\int_{\Omega} \Phi \frac{\partial x}{\partial n} R \mathrm{~d} x \mathrm{~d} y=-\int_{\Omega} \Phi \eta_{x} \mathrm{~d} x \mathrm{~d} y,
\end{aligned}
$$

because no net contribution is made by surface integrals of $\Phi \partial x / \partial n$ over the other sections of the boundary of $\mathscr{D}$. A corresponding reduction holds for the integral of $\phi_{x}^{\prime}{ }_{x}$ over $\mathscr{D}^{\prime}$, whence addition of the expressions for the two parts of $K$ gives (3.2).

The interpretation of $K$ needs careful qualification, however, in the case of a 
localized motion for which $\Omega=\mathbb{R} \times \mathbb{R}$. Using spherical coordinates $(r, \theta, \psi)$ and referring Green's theorem to $\mathscr{D}$ bounded above by $S$ and below by a hemisphere of radius $r \rightarrow \infty$, we get

$$
\begin{aligned}
\int_{\mathscr{D}} \phi_{x} \mathrm{~d} x \mathrm{~d} y \mathrm{~d} z & =-\int_{\Omega} \Phi \eta_{x} \mathrm{~d} x \mathrm{~d} y+\lim _{r \rightarrow \infty} \int_{-\pi}^{0} \int_{0}^{\pi} \phi \cos \theta r^{2} \sin \theta \mathrm{d} \theta \mathrm{d} \psi \\
& =-\int_{\Omega} \Phi \eta_{x} \mathrm{~d} x \mathrm{~d} y+\frac{2}{3} \pi C,
\end{aligned}
$$

where $C$ is the coefficient of the dipole far-field aligned with the $x$-axis (i.e. $\phi=$ $C r^{-2} \cos \theta+O\left(r^{-3}\right)$ as $\left.r \rightarrow \infty\right)$. Similarly,

$$
\int_{\mathscr{Q}^{\prime}} \phi_{x}^{\prime} \mathrm{d} x \mathrm{~d} y \mathrm{~d} z=\int_{\Omega} \Phi^{\prime} \eta_{x} \mathrm{~d} x \mathrm{~d} y+\frac{2}{3} \pi C^{\prime} .
$$

Thus the term $\frac{2}{3} \pi\left(\rho U C+\rho^{\prime} U^{\prime} C^{\prime}\right)$ should be added on the right-hand side of (3.2) to provide $K$ with a literal interpretation as the kinetic-energy perturbation. (For two-dimensional motions independent of $y$, the coefficient $C$ is defined by $\phi=$ $C x / r^{2}+O\left(r^{-2}\right)$ as $r=\left(x^{2}+z^{2}\right)^{1 / 2} \rightarrow \infty$; and $C^{\prime}$ is defined similarly. The addition to the respective version of (3.2) is consequently $\frac{1}{2} \pi\left(\rho U C+\rho^{\prime} U^{\prime} C^{\prime}\right)$.)

The question therefore arises whether $C$ and $C^{\prime}$ can be non-zero in the modelling of realistic localized motions. For the water-wave problem (i.e. the special case $\rho^{\prime}=0$ ) there is good reason to exclude the possibility of a dipole far field, because it would imply infinite angular momentum in the half-space $D$; a motion with this feature therefore cannot be created by finite forces (see Benjamin \& Olver 1982, p. 175). In the present model the total angular momentum is easily seen to be infinite unless $\rho C=\rho^{\prime} C^{\prime}$, which condition ensures that equal and opposite angular momenta occur in $\mathscr{D}$ and $\mathscr{D}^{\prime}$ although both are infinite if $C \neq 0$ (cf. Benjamin \& Olver, footnote to p. 175). This condition is also implied by (2.2), which shows asymptotically that $\rho C_{t}=\rho^{\prime} C^{\prime}{ }_{t}$ during the application of external forces over a finite part of $S$ to initiate a motion. The creation of dipole far fields appears to be an extraordinary possibility for our model, however, and no significant behaviour appears to be missed by the assumption that $C=C^{\prime}=0$. Under the assumption, of course, the identification of the expression (3.2) with the kinetic-energy perturbation is unequivocal.

Now, respective to an arbitrary fixed $\eta=\eta(x, y)$, taken to be sufficiently smooth, consider the class $\mathscr{C}$ of pairs of functions $\chi \in \mathrm{H}^{1}(\mathscr{D}), \chi^{\prime} \in \mathrm{H}^{1}\left(\mathscr{D}^{\prime}\right)$, and write $X(x, y)=\chi(x, y, \eta(x, y)), X^{\prime}=\chi^{\prime}(x, y, \eta(x, y))$. (Here $\mathrm{H}^{1} \equiv \mathrm{W}^{1,2}$ is the Sobolev space of measurable functions with square-integrable generalized derivatives in the respective domain. The foregoing attribution implies that $X, X^{\prime} \in \mathrm{H}^{1 / 2}(\Omega)$ (cf. Adams 1975) and so these functions are absolutely integrable over $\Omega$.) The following functional is well defined in the class $\mathscr{C}$ :

$$
\begin{aligned}
\mathscr{K}\left(\chi, \chi^{\prime}\right) & =-\int_{\Omega}\left(\rho U X-\rho^{\prime} U^{\prime} X^{\prime}\right) \eta_{x} \mathrm{~d} x \mathrm{~d} y \\
& +\int_{\mathscr{D}} \frac{1}{2} \rho|\nabla \chi|^{2} \mathrm{~d} x \mathrm{~d} y \mathrm{~d} z+\int_{\mathscr{D}^{\prime}} \frac{1}{2} \rho^{\prime}\left|\nabla \chi^{\prime}\right|^{2} \mathrm{~d} x \mathrm{~d} y \mathrm{~d} z .
\end{aligned}
$$

Accordingly, for a given function $\zeta=\zeta(x, y)$ supposed sufficiently smooth (more generally for $\zeta \in \mathrm{H}^{1 / 2}(\Omega)$ ), we may define

$$
K(\zeta)=\min _{\rho X-\rho^{\prime} X^{\prime}=\zeta} \mathscr{K}\left(\chi, \chi^{\prime}\right) .
$$


Being convex, the functional $\mathscr{K}$ has a unique minimizer $\left(\phi, \phi^{\prime}\right)$ in the class $\mathscr{C}$ under the linear constraint $\rho X-\rho^{\prime} X^{\prime}=\zeta$.

The first variation $\dot{\mathscr{K}}$ of $\mathscr{K}$ relative to the minimum is given by putting $\chi=\phi+\dot{\chi}$, $\chi^{\prime}=\phi^{\prime}+\dot{\chi}^{\prime}$ in (3.3) and linearizing in $\dot{\chi}$ and $\dot{\chi}^{\prime}$. Using the weak (distributional) form of Green's theorem, we obtain

$$
\begin{aligned}
\dot{\mathscr{K}}= & -\int_{\Omega}\left(\rho U \dot{X}-\rho^{\prime} U^{\prime} \dot{X}^{\prime}\right) \eta_{x} \mathrm{~d} x \mathrm{~d} y+\int_{\mathscr{D}} \rho \nabla \phi \cdot \nabla \dot{\chi} \mathrm{d} x \mathrm{~d} y \mathrm{~d} z+\int_{\mathscr{D}^{\prime}} \rho^{\prime} \nabla \phi^{\prime} \cdot \nabla \dot{\chi}^{\prime} \mathrm{d} x \mathrm{~d} y \mathrm{~d} z \\
= & \int_{\Omega}\left\{\rho \dot{X}\left(R \Phi_{(n)}-U \eta_{x}\right)-\rho^{\prime} \dot{X}^{\prime}\left(R \Phi_{(n)}^{\prime}-U^{\prime} \eta_{x}\right)\right\} \mathrm{d} x \mathrm{~d} y \\
& -\int_{\mathscr{D}} \rho \dot{\chi} \Delta \phi \mathrm{d} x \mathrm{~d} y \mathrm{~d} z-\int_{\mathscr{D}^{\prime}} \rho^{\prime} \dot{\chi}^{\prime} \Delta \phi^{\prime} \mathrm{d} x \mathrm{~d} y \mathrm{~d} z,
\end{aligned}
$$

which must vanish for arbitrary $\dot{\chi}$ and $\dot{\chi}^{\prime}$. Thus (3.5) verifies the following properties of the minimizer $\left(\phi, \phi^{\prime}\right)$ for given $\zeta$ and $\eta$ : (i) $\Delta \phi=0$ in $\mathscr{D}$ and $\Delta \phi^{\prime}=0$ in $\mathscr{D}^{\prime}$; (ii) because $\rho \dot{X}=\rho^{\prime} \dot{X}^{\prime}$ (owing to the linear constraint in (3.4)),

$$
R \Phi_{(n)}-U \eta_{x}=R \Phi_{(n)}^{\prime}-U^{\prime} \eta_{x} .
$$

Note that the property (3.6), as required by (2.1), is thus automatically provided by the variational characterization (3.4) of $K(\zeta)$. Although equivalent, it would be comparatively awkward to define $\phi$ and $\phi^{\prime}$ a priori as harmonic functions in $\mathscr{D}$ and $\mathscr{D}^{\prime}$ satisfying (3.6), $\rho \Phi-\rho^{\prime} \Phi^{\prime}=\zeta$ and respective asymptotic conditions for $z \rightarrow \pm \infty$.

Again for fixed $\eta$, let us next express the first (infinitesimal) variation $\dot{K}$ of $K$ corresponding to $\dot{\zeta}=\rho \dot{\Phi}-\rho^{\prime} \dot{\Phi}^{\prime}$. By virtue of Green's theorem, the result from (3.2) is

$$
\dot{K}=\int_{\Omega}\left\{\rho \dot{\Phi}\left(R \Phi_{(n)}-U \eta_{x}\right)-\rho^{\prime} \dot{\Phi}^{\prime}\left(R \Phi^{\prime}{ }_{(n)}-U^{\prime} \eta_{x}\right)\right\} \mathrm{d} x \mathrm{~d} y,
$$

which in view of (3.6) shows that the variational derivative of $K$ with respect to $\zeta$ is

$$
\frac{\delta K}{\delta \zeta}=R \Phi_{(n)}-U \eta_{x}=R \Phi_{(n)}^{\prime}-U^{\prime} \eta_{x} .
$$

It remains to examine the first variation of $K$ including the contribution by infinitesimal variations $\dot{\eta}$ of $\eta$. We note that

$$
\dot{\Phi}=\dot{\phi}(x, y, \eta)+\phi_{z}(x, y, \eta) \dot{\eta}=(\dot{\phi})_{S}+\Phi_{(z)} \dot{\eta},
$$

say, and similarly

$$
\dot{\Phi}^{\prime}=\left(\dot{\phi}^{\prime}\right)_{S}+\Phi_{(z)}^{\prime} \dot{\eta} .
$$

In the evaluation of $\dot{K}$ from (3.2), the integrals over $\mathscr{D}$ and $\mathscr{D}^{\prime}$ evidently contribute a term expressible as the integral of $\left(\frac{1}{2} \rho q^{2}-\frac{1}{2} \rho^{\prime} q^{\prime 2}\right) \dot{\eta}$ over $\Omega$, together with terms arising from the differences between $\dot{\Phi}$ and $(\dot{\phi})_{S}, \dot{\Phi}^{\prime}$ and $\left(\phi^{\prime}\right)_{S}$. It is thus found that

$$
\frac{\delta K}{\delta \eta}=\rho\left(\frac{1}{2} q^{2}-\Phi_{(z)} R \Phi_{(n)}+U \Phi_{x}\right)-\rho^{\prime}\left(\frac{1}{2} q^{\prime 2}-\Phi_{(z)}^{\prime} R \Phi_{(n)}^{\prime}+U^{\prime} \Phi^{\prime}{ }_{x}\right) .
$$

\subsection{The Hamiltonian equations}

Depending on $\eta$ and $\zeta$ alone, the Hamiltonian functional is the total energy $E=K+V$, where

$$
V=\int_{\Omega}\left\{\frac{1}{2} g\left(\rho-\rho^{\prime}\right) \eta^{2}+\sigma(R-1)\right\} \mathrm{d} x \mathrm{~d} y
$$


is potential energy relative to the undisturbed state of the model system. The second component of $V$ is superficial energy, namely surface tension $\sigma$ times the excess surface area of the disturbed interface. It follows from (3.9) that

$$
\frac{\delta V}{\delta \eta}=g\left(\rho-\rho^{\prime}\right) \eta-2 \sigma \mathrm{H}
$$

and obviously $\delta V / \delta \zeta=0$.

For comparison with the dynamical condition (2.2) satisfied at the interface, note that

$$
\Phi_{t}=\Phi_{(t)}+\Phi_{(z)} \eta_{t}, \quad \Phi_{x}=\Phi_{(x)}+\Phi_{(z)} \eta_{x}
$$

and similarly for $\Phi_{t}^{\prime}$ and $\Phi^{\prime}{ }_{x}$. Hence, in view of (3.7), (3.8) and (3.10), the evolutionary system consisting of (2.1) and (2.2) is seen to be represented by

$$
\eta_{t}=\frac{\delta E}{\delta \zeta}, \quad \zeta_{t}=-\frac{\delta E}{\delta \eta},
$$

which exemplifies canonical Hamiltonian structure (cf. Benjamin \& Olver 1982, equation (2.8), to which the present system reduces in all respects when $\rho^{\prime}=0$ and $U=0)$.

It deserves emphasis that the same representation (3.11) of the full dynamical problem applies for each specification, as appropriate, of the horizontal domain $\Omega$. This representation also applies when the liquids are given rigid boundaries at finite distance but disjoint from the interface $S$. Then $\mathscr{D}$ and $\mathscr{D}^{\prime}$ in the expressions (3.2) for $K$ and (3.3) for $\mathscr{K}$ are prescribed accordingly, and the conditions $\partial \phi / \partial n=0$ and $\partial \phi^{\prime} / \partial n=0$ at the respective solid boundaries are satisfied as additional intrinsic properties of the minimizer $\left(\phi, \phi^{\prime}\right)$ for the variational principle (3.4).

\subsection{Symmetries and conservation laws}

In the case $U=U^{\prime}=0$ the present model describes free interfacial waves; and then the symmetry group for the three-dimensional problem (3.11) is an obvious modification of the symmetry group for the water-wave problem $\left(\rho^{\prime}=0\right)$, which when surface tension is operative is composed of twelve one-parameter subgroups (Benjamin \& Olver, Theorem 4.2). Eleven conservation laws can then be found corresponding to those for the water-wave problem (Benjamin \& Olver, Theorem 5.3). Various symmetries are lost, however, when $U \neq U^{\prime}$ : in particular, the horizontal rotation subgroup and the subgroup named scaled (vertical) acceleration by Benjamin \& Olver (1982, p. 151). The number of conservation laws is also reduced when $U \neq U^{\prime}$, although all the simpler, more informative ones survive. We shall not cover these aspects comprehensively here, merely noting as follows the most conspicuous symmetries and consequent conservative properties.

First consider translation along the $t$-axis, which is a symmetry if $g$ is independent of $t$ so that the system (3.11) is autonomous. It then follows that $E=$ const. along any trajectory of the Hamiltonian system, because

$$
\frac{\mathrm{d} E}{\mathrm{~d} t}=\left\langle\frac{\delta E}{\delta \eta}, \eta_{t}\right\rangle+\left\langle\frac{\delta E}{\delta \zeta}, \zeta_{t}\right\rangle=\left\langle\frac{\delta E}{\delta \eta}, \frac{\delta E}{\delta \zeta}\right\rangle-\left\langle\frac{\delta E}{\delta \zeta}, \frac{\delta E}{\delta \eta}\right\rangle=0 .
$$

Here $\langle\cdot, \cdot\rangle$ denotes an $\mathrm{L}_{2}$ inner product in respect of integration over $\Omega$. If $g$ varies with $t$, the representation (3.11) of the dynamical problem still holds; but then $\mathrm{d} E / \mathrm{d} t=\frac{1}{2}(\mathrm{~d} g / \mathrm{d} t)\left(\rho-\rho^{\prime}\right)\left\langle\eta^{2}, 1\right\rangle$.

For fixed $g$, the constant value of $E$ may be positive, negative or zero, and this 
feature has an important bearing on the interpretation of possible motions. In common with all other Hamiltonian systems, the following property is easily confirmable by the linearized theory summarized at the end of $\$ 2$ (see $\$ 5$ for details). The onset of instability with $|\operatorname{Im}(s)|>0$ (i.e. the occurrence of an oscillatory wave mode with exponentially growing amplitude) arises from the coincidence of wave modes with $E>0$ and $E<0$ respectively as the limit of stability is approached. Furthermore, any disturbance growing to finite size from an infinitesimal neighbourhood of the undisturbed state must be characterized by $E=0$. Kelvin-Helmholtz instability is well known to develop into huge deformations of the interface, going beyond the reach of the foregoing theory which is delimited by the assumption that $\eta$ remains a single-valued function of $(x, y)$; but the characterization $E=0$ holds throughout all stages of such motions (see $\S 4$ ).

The facts that the vector-valued function $-\left[\eta_{t}, \zeta_{t}\right]^{T}$ represents the infinitesimal generator of the time-translation subgroup and that the invariance of $E$ follows from (3.11) exemplify a general rule for identifying the invariants of a Hamiltonian system (cf. Benjamin \& Olver 1982, §5). For the same reason, because horizontal translations are represented by $-\left[\eta_{x}, \zeta_{x}\right]^{T}$ and $-\left[\eta_{y}, \zeta_{y}\right]^{T}$, the invariance of functionals $I_{1}$ and $I_{2}$ is ensured by their satisfying

$$
-\left(\begin{array}{c}
\eta_{x} \\
\zeta_{x}
\end{array}\right)=\boldsymbol{J} \operatorname{grad} I_{1}, \quad-\left(\begin{array}{c}
\eta_{y} \\
\zeta_{y}
\end{array}\right)=\boldsymbol{J} \operatorname{grad} I_{2},
$$

where $\boldsymbol{J}$ is the skew-symmetric matrix implicit in (3.11), namely

$$
\boldsymbol{J}=\left(\begin{array}{cc}
0 & 1 \\
-1 & 0
\end{array}\right), \quad \text { and } \operatorname{grad} I_{j}=\left(\begin{array}{l}
\delta I_{j} / \delta \eta \\
\delta I_{j} / \delta \zeta
\end{array}\right) \quad(j=1,2) \text {. }
$$

From (3.12) it can be seen at once that

$$
I_{1}=-\int_{\Omega} \zeta \eta_{x} \mathrm{~d} x \mathrm{~d} y, \quad I_{2}=-\int_{\Omega} \zeta \eta_{y} \mathrm{~d} x \mathrm{~d} y,
$$

and these invariant functionals are describable as the components of horizontal impulse in the $x$-direction and $y$-direction respectively.

An interesting variational principle hence follows for the case of two-dimensional motions independent of $y$. A steady wave motion in this class is a non-trivial solution of (3.11) in the form $\eta(x-c t), \zeta(x-c t)$, where the phase velocity $c$ is a real constant. Because $\partial_{t}=-c \partial_{x}$ as an operation on such a solution, comparison between (3.11) and the first of (3.12) shows that

$$
\operatorname{grad} E=c \operatorname{grad} I_{1} .
$$

Thus any such solution is a conditional extremal of $E$ subject to $I_{1}$ being prescribed, and $c$ is the Lagrange multiplier. The variational principle serves particularly to characterize steady periodic waves, competitors for a conditional extremum of $E$ being pairs of periodic functions with a given period; and plainly, since both $E$ and $I$ are invariants of any free motion, a conditional minimum implies that the steady wave motion is orbitally stable to disturbances of the same period (i.e. Lyapunov stable in respect of a translation-invariant metric). A corresponding variational principle has long been known to apply to steady water waves (Benjamin 1972, Appendix; 1974, $\S 5 ; 1984, \S 6.3)$; and the extension to unforced interfacial waves $\left(U=U^{\prime}=0\right)$ is hardly surprising, although it has not been noticed previously as far as we know. On the other hand, the extension of the variational principle to the Kelvin-Helmholtz 
problem is somewhat surprising at first, and we shall need to examine its implications carefully in $\S 5$.

A few other symmetries and conservative properties will be noted in $\S 4.3$.

\subsection{Alternative Hamiltonian formulation}

For two-dimensional wave motions there is marginal advantage in the following alternative representation, which adapts the approach to the water-wave problem proposed by Benjamin $(1984, \mathrm{Ch} .5)$. Introduce the stream functions $\psi$ and $\psi^{\prime}$, which are the harmonic conjugates of $\phi$ and $\phi^{\prime}$ in $\mathscr{D}$ and $\mathscr{D}^{\prime}$ respectively, satisfying $\phi_{x}=\psi_{z}$, $\phi_{z}=-\psi_{x}$ and $\phi_{x}^{\prime}=\psi_{z}^{\prime}, \phi_{z}^{\prime}=-\psi^{\prime}{ }_{x}$. The kinematic conditions (2.1) at the interface become

$$
\eta_{t}+(U \eta+\Psi)_{x}=0, \quad \eta_{t}+\left(U^{\prime} \eta+\Psi^{\prime}\right)_{x}=0,
$$

where $\Psi=\psi(x, \eta, t)$ and $\Psi^{\prime}=\psi^{\prime}(x, \eta, t)$. Expressing $K$ in terms of $\psi$ and $\psi^{\prime}$ rather than as in (3.2), defining a respective convex functional over $\mathrm{H}^{1}(\mathscr{D}) \times \mathrm{H}^{1}\left(\mathscr{D}^{\prime}\right)$ akin to $\mathscr{K}$ defined by (3.3), and recognizing $K$ to be the unique minimizer of this functional subject to the linear constraint

$$
\left(\rho \Psi_{(n)}-\rho^{\prime} \Psi_{(n)}^{\prime}\right) R=\zeta_{x}=W,
$$

say, one finds that

$$
U \eta+\Psi=U^{\prime} \eta+\Psi^{\prime}
$$

is automatically a property of the minimizer $\left(\psi, \psi^{\prime}\right)$. Furthermore, the equal expressions on each side of (3.16) are found to be the same as $\delta K / \delta W$. Hence (3.15) and the dynamical equation (2.2) are seen to be recovered by

$$
\eta_{t}+(\delta E / \delta W)_{x}=0, \quad W_{t}+(\delta E / \delta \eta)_{x}=0
$$

(cf. Benjamin 1984, equation (6.5)).

Like (3.11), being indeed equivalent to it, the system (3.17) is Hamiltonian: the cosymplectic skew-symmetric operator in the condensed representation

$$
\left[\eta_{t}, W_{t}\right]^{T}=\boldsymbol{J} \operatorname{grad} E \quad \text { is } \quad \boldsymbol{J}=\left(\begin{array}{cc}
0 & -\partial_{x} \\
-\partial_{x} & 0
\end{array}\right) .
$$

A comparative merit of (3.17) is that both equations have the form of conservation laws. This feature may be helpful in the case that the fluids have at least one horizontal rigid boundary and long-wave approximations need to be developed (cf. Benjamin 1984, §6.5). Another merit is that, according to the rule exemplified by the first of (3.12), the impulse invariant is even more evidently than before given by $I_{1}=\langle\eta, W\rangle$.

In this comparatively simple case of two-dimensional motions, it is of interest to find explicitly the local conservation law that is implied by the invariance of the impulse functional $I_{1}$. Because $I_{1}$ is the integral of the density $\eta W$ over $\Omega$, we can presume the existence of a flux density $S$ (called flow force in Benjamin 1984, where the general role of such fluxes in impulse-conservation laws for Hamiltonian systems was discussed at length) that satisfies

$$
(\eta W)_{t}+S_{x}=0
$$

everywhere in $\Omega$. The corresponding flux for the two-dimensional water-wave problem is already on record, having a surprising form (Benjamin 1984, §6.4). By substitution 
from (3.17) for the time derivatives of $\eta$ and $W$, we obtain from (3.18)

$$
\begin{aligned}
S_{x} & =-\eta W_{t}-\eta_{t} W \\
& =\eta(\delta E / \delta \eta)_{x}+W(\delta E / \delta W)_{x} \\
& =(\eta \delta E / \delta \eta)_{x}-(\delta \mathrm{E} / \delta \eta) \eta_{x}+W(U \eta+\Psi)_{x} .
\end{aligned}
$$

Here the alternative $U^{\prime} \eta+\Psi^{\prime}$ for $\delta E / \delta W$ according to (3.16) can be used in the last term.

To evaluate $\delta E / \delta \eta$ in this expression for $S_{x}$, the results (3.8) and (3.10) are available, (3.8) needing to be reformulated in terms of the derivatives of $\psi$ and $\psi^{\prime}$ at the interface: for example, $\Phi_{(z)}=-\Psi_{(x)}$ and

$$
R \Phi_{(n)}=\Phi_{(z)}-\eta_{x} \Phi_{(x)}=-\Psi_{(x)}-\eta_{x} \Psi_{(z)}=-\Psi_{x},
$$

because $R=\left(1+\eta_{x}^{2}\right)^{1 / 2}$. It is thus found that

$$
\begin{aligned}
S_{x}=( & \eta \delta E / \delta \eta)_{x}+W(U \eta+\Psi)_{x}-\eta_{x}\left\{g\left(\rho-\rho^{\prime}\right) \eta-\sigma \eta_{x x} R^{-3}\right\} \\
& \quad-\eta_{x}\left\{\rho\left(\frac{1}{2} q^{2}-\Psi_{(x)} \Psi_{x}+U R \Psi_{(n)}\right)-\rho^{\prime}\left(\frac{1}{2} q^{\prime 2}-\Psi^{\prime}{ }_{(x)} \Psi^{\prime}{ }_{x}+U^{\prime} R \Psi^{\prime}{ }_{(n)}\right)\right\},
\end{aligned}
$$

whence (3.16) and the definition of $W$ above (3.16) show that various terms cancel and there follows

$$
\begin{aligned}
S_{x}= & (\eta \delta E / \delta \eta)_{x}+\rho \Psi_{(z)} \Psi_{(x)}-\rho^{\prime} \Psi_{(z)}^{\prime} \Psi_{(x)}^{\prime} \\
& +\eta_{x}\left\{\frac{1}{2} \rho\left(\Psi_{(z)}^{2}-\Psi_{(x)}^{2}\right)-\frac{1}{2} \rho^{\prime}\left(\Psi_{(z)}^{\prime 2}-\Psi_{(x)}^{\prime 2}\right)-g\left(\rho-\rho^{\prime}\right) \eta+\sigma \eta_{x x} R^{-3}\right\} .
\end{aligned}
$$

Hence we conclude that

$$
\begin{aligned}
S=\eta \delta E & / \delta \eta-\frac{1}{2} g\left(\rho-\rho^{\prime}\right) \eta^{2}-\sigma R^{-1} \\
& +\int_{-\infty}^{\eta} \frac{1}{2} \rho\left(\psi_{z}^{2}-\psi_{x}^{2}\right) \mathrm{d} z+\int_{\eta}^{\infty} \frac{1}{2} \rho^{\prime}\left({\psi^{\prime}}_{z}^{2}-\psi_{x}^{\prime 2}\right) \mathrm{d} z .
\end{aligned}
$$

Partial differentiation of this expression with respect to $x$ is readily seen to recover (3.19). The terms proportional to $\eta_{x}$ follow immediately. Differentiation of the two integrals also gives integrals, the first two terms of which can be integrated by parts, recovering the second and third terms on the right-hand side of (3.19), and the remainder is zero because $\Delta \psi=0$ in $\mathscr{D}$ and $\Delta \psi^{\prime}=0$ in $\mathscr{D}^{\prime}$.

For a steady wave motion described by a solution of (3.17) in the form $\eta(x-c t)$, $W(x-c t)$, where the phase velocity $c$ is a real constant, (3.18) implies that

$$
\bar{S}=S-c(\eta W)
$$

is independent of $x$. (Steady-wave solutions of the nonlinear Kelvin-Helmholtz problem will be investigated in Part 2.) Such a solution satisfies the variational principle (3.14), which implies in particular that $\delta E / \delta \eta=c \delta I_{1} / \delta \eta=c W$. Thus the first term on the right-hand side of (3.20) is cancelled in $\bar{S}$ given by (3.21), and we have

$$
\begin{aligned}
\bar{S}=-\frac{1}{2} g(\rho & \left.-\rho^{\prime}\right) \eta^{2}-\sigma R^{-1} \\
& +\int_{-\infty}^{\eta} \frac{1}{2} \rho\left(\psi_{z}^{2}-\psi_{x}^{2}\right) \mathrm{d} z+\int_{\eta}^{\infty} \frac{1}{2} \rho^{\prime}\left(\psi_{z}^{\prime 2}-\psi_{x}^{\prime 2}\right) \mathrm{d} z
\end{aligned}
$$

(cf. Benjamin 1984, equation (6.9), the corresponding result for the water-wave problem in the absence of surface tension).

The result (3.22) is doubly remarkable, first because it has no explicit dependence 
on $c$ and secondly because, in common with (3.20), it has no explicit dependence on $U$ and $U^{\prime}$. One may readily confirm directly, however, that $\bar{S}$ is constant for any steady-wave solution of the system (3.17), which for steady waves reduces to

$$
(c-U) \eta=\Psi, \quad c W=\delta E / \delta \eta .
$$

Note that $S$ and $\bar{S}$ are fully determined by the dependent variables $\eta$ and $W$, but not by the local values of them and their derivatives qua functions of $x$. This limitation is inherent because the system of evolutionary equations (3.17) entails nonlocal operators (e.g. $\Psi$ in $\delta E / \delta W=U \eta+\Psi$ is evidently a non-local transformation of $W$ and $\eta$ ).

The generalization of (3.17) to three-dimensional motions requires the threefold set of dependent variables $\eta, \eta_{x}$ and $\eta_{y}$. The method has been explained by Benjamin $(1984, \S 6.6)$, but it offers little advantage over the simpler, two-component formulation (3.11).

\section{Hamiltonian formulation when the interface is folded}

Later stages in the evolution from Kelvin-Helmholtz instability have been investigated mainly by numerical solution of the hydrodynamic problem - or its approximate simulation by a line of discrete vortices (e.g. see Peltier, Hallé \& Clark 1978; Aref \& Siggia 1980; Higdon \& Pozrikidis 1985; Klaassen \& Peltier 1985). The usual model is two-dimensional and periodic in $x$. The interface constituting a vortex sheet has been found to become folded, eventually rolling up into a spiralling billow. Another example where a parametric representation is necessary is the overhanging interfacial waves found by Meiron \& Saffman (1983). Our aim now is to demonstrate the Hamiltonian structure that holds throughout all such developments. The needed parametric representation of the interface is closely related to that for water waves, as explained by Benjamin \& Olver (1982, Appendix 1), and to that for bubbles as explained by Benjamin (1987).

\subsection{Parametric representation of $S$}

The axes used in $\S 2$ and $\S 3$ are now relabelled $\left(x_{1}, x_{2}, x_{3}\right) \stackrel{\text { def }}{=}(x, y, z)$, and the interface $S$ is taken to be described parametrically by

$$
x_{i}=X_{i}(\mu, v, t), \quad i=1,2,3
$$

where $(\mu, v)$ ranges over a two-dimensional domain $\Omega$. We write

$$
\Phi=\phi_{S} \stackrel{\text { def }}{=} \phi\left(X_{1}, X_{2}, X_{3}, t\right), \quad \Phi^{\prime}=\phi_{S}^{\prime}=\phi^{\prime}\left(X_{1}, X_{2}, X_{3}, t\right)
$$

and, further compressing the notation for spatial derivatives of $\phi$ and $\phi^{\prime}$ evaluated at $S$,

In terms of

$$
\Phi_{(i)}=\left(\frac{\partial \phi}{\partial x_{i}}\right)_{S} \quad \text { and } \quad \Phi_{(i)}^{\prime}=\left(\frac{\partial \phi^{\prime}}{\partial x_{i}}\right)_{S}
$$

$$
\gamma_{1}=\frac{\partial\left(X_{2}, X_{3}\right)}{\partial(\mu, v)}, \quad \gamma_{2}=\frac{\partial\left(X_{3}, X_{1}\right)}{\partial(\mu, v)}, \quad \gamma_{3}=\frac{\partial\left(X_{1}, X_{2}\right)}{\partial(\mu, v)}, \quad J=\left(\gamma_{i}^{2}\right)^{1 / 2},
$$

the components of the unit normal to $S$ directed into the less-dense liquid are $\gamma_{i} / J$, 
and the element of surface area on $S$ is $J \mathrm{~d} \mu \mathrm{d} \nu$. (Note that, in the above definition of $J$ and henceforth, repeated subscripts imply summation over $1,2,3$.)

The kinematic conditions (2.1), relating the normal velocity of $S$ to that in the contiguous liquids, must now be replaced by their generalizations

$$
\left.\begin{array}{l}
\gamma_{i} \partial_{t} X_{i}-U \gamma_{1}=J \Phi_{(n)}=\gamma_{i} \Phi_{(i)}, \\
\gamma_{i} \partial_{t} X_{i}-U^{\prime} \gamma_{1}=J \Phi_{(n)}^{\prime}=\gamma_{i} \Phi_{(i)}^{\prime} .
\end{array}\right\}
$$

Correspondingly, because

$$
\partial_{t} \Phi=\Phi_{(t)}+\Phi_{(i)} \partial X_{i} / \partial t
$$

and similarly for $\partial \Phi^{\prime} / \partial t$, the dynamical condition (2.2) is generalized by

$$
\begin{aligned}
\zeta_{t}+ & \rho\left(U \Phi_{(1)}+\frac{1}{2} q^{2}-\Phi_{(i)} \partial_{t} X_{i}\right) \\
& \quad-\rho^{\prime}\left(U^{\prime} \Phi^{\prime}{ }_{(1)}+\frac{1}{2} q^{\prime 2}-\Phi_{(i)}^{\prime} \partial_{t} X_{i}\right)=-g\left(\rho-\rho^{\prime}\right) X_{3}+2 \sigma H,
\end{aligned}
$$

in which $\zeta=\rho \Phi-\rho^{\prime} \Phi^{\prime}$ as before and $q^{2}=\Phi_{(i)}^{2}, q^{\prime 2}=\Phi_{(i)}^{\prime 2}$. The mean curvature $H$ of the surface $S$ can be expressed in the standard way as a nonlinear function of the six derivatives $\partial X_{i} / \partial \mu$ and $\partial X_{i} / \partial v$, but there is no need to spell out this detail here. (It will suffice for present purposes merely to recognize another well-known result from differential geometry, that $-2 H \gamma_{i}$ is the variational derivative of the surface-area integral with respect to $X_{i}$; see (4.4) and (4.5) below.)

\subsection{The Hamiltonian equations}

Turning to the generalization of the total-energy functional, let us consider potential energy as originally expressed by (3.9). It is now given by

$$
V=\int_{\Omega}\left\{\frac{1}{2} g\left(\rho-\rho^{\prime}\right) X_{3}^{2} \gamma_{3}+\sigma\left(J-\gamma_{3}\right)\right\} \mathrm{d} \mu \mathrm{d} \nu
$$

which of course reduces to (3.9) in the restricted case $X_{1}=\mu=x_{1}, X_{2}=v=x_{2}$, $X_{3}=\eta\left(x_{1}, x_{2}, t\right)$. Variational derivatives are now defined by the $\mathrm{L}_{2}$ inner product corresponding to integration with respect to $(\mu, v)$ over a fixed domain, denoted as before by $\Omega$. It is accordingly found from (4.4) that

$$
\frac{\delta V}{\delta X_{i}}=\left\{g\left(\rho-\rho^{\prime}\right) X_{3}-2 \sigma H\right\} \gamma_{i}
$$

Generalizing (3.2), an expression for the kinetic-energy perturbation is

$$
\begin{aligned}
K=\int_{\Omega}( & \left.\rho U \Phi-\rho^{\prime} U^{\prime} \Phi^{\prime}\right) \gamma_{1} \mathrm{~d} \mu \mathrm{d} v \\
& +\int_{\mathscr{D}} \frac{1}{2} \rho|\nabla \phi|^{2} \mathrm{~d} x_{1} \mathrm{~d} x_{2} \mathrm{~d} x_{3}+\int_{\mathscr{D}^{\prime}} \frac{1}{2} \rho^{\prime}\left|\nabla \phi^{\prime}\right|^{2} \mathrm{~d} x_{1} \mathrm{~d} x_{2} \mathrm{~d} x_{3},
\end{aligned}
$$

which entails no restriction on the curved boundary $S$ of $\mathscr{D}$ and $\mathscr{D}^{\prime}$. Proceeding as in $\$ 3$ to identify $K$, for given $S$, as the unique minimum of this functional defined over $\mathrm{H}^{1}(\mathscr{D}) \times \mathrm{H}^{1}\left(\mathscr{D}^{\prime}\right)$, we deduce in the same way as before that

$$
U \gamma_{1}+\gamma_{i} \Phi_{(i)}=U^{\prime} \gamma_{1}+\gamma_{i} \Phi_{(i)}^{\prime}=\frac{\delta K}{\delta \zeta} .
$$

Recognizing that

$$
\dot{\zeta}=\rho\left\{(\dot{\Phi})_{S}+\Phi_{(i)} \dot{X}_{i}\right\}-\rho^{\prime}\left\{\left(\dot{\Phi}^{\prime}\right)_{S}+\Phi_{(i)}^{\prime} \dot{X}_{i}\right\}
$$


we find further that

$$
\begin{aligned}
\frac{\delta K}{\delta X_{i}}=\rho( & \left.\frac{1}{2} q^{2} \gamma_{i}-\Phi_{(i)} \gamma_{j} \Phi_{(j)}\right)-\rho^{\prime}\left(\frac{1}{2} q^{\prime 2} \gamma_{i}-\Phi_{(i)}^{\prime} \gamma_{j} \Phi_{(j)}^{\prime}\right) \\
& +\left(\rho U \Phi_{(1)}-\rho^{\prime} U^{\prime} \Phi_{(1)}^{\prime}\right) \gamma_{i}-\left(\rho U \Phi_{(i)}-\rho^{\prime} U^{\prime} \Phi_{(i)}^{\prime}\right) \gamma_{1},
\end{aligned}
$$

where the terms in the second line, deriving from the first integral in (4.6), make a contribution only for $i=2,3$.

Now, the solution of the evolutionary problem may be considered as the vectorvalued function

$$
\boldsymbol{Z}=\left[X_{1}, X_{2}, X_{3}, \zeta\right]^{T}=\boldsymbol{Z}(\mu, v, t),
$$

and our aim is to establish a Hamiltonian formulation of the problem in terms of $\boldsymbol{Z}$. Writing

we define

$$
\zeta_{(i)}=\rho \Phi_{(i)}-\rho^{\prime} \Phi_{(i)}^{\prime}, \quad i=1,2,3,
$$

and the skew-symmetric matrix

$$
a_{i j}=\gamma_{i} \zeta_{(j)}-\gamma_{j} \zeta_{(i)}=-a_{j i}
$$

$$
\boldsymbol{M}(\boldsymbol{Z})=\left[\begin{array}{cccc}
0 & a_{12} & a_{13} & -\gamma_{1} \\
a_{21} & 0 & a_{23} & -\gamma_{2} \\
a_{31} & a_{32} & 0 & -\gamma_{3} \\
\gamma_{1} & \gamma_{2} & \gamma_{3} & 0
\end{array}\right]
$$

The system (4.2) and (4.3) is hence seen, in view of (4.5), (4.7) and (4.8), to be equivalent to

$$
\boldsymbol{M}(\boldsymbol{Z})\left(\partial_{t} \boldsymbol{Z}\right)=\operatorname{grad} E(\boldsymbol{Z}),
$$

where $E=K+V$ and $\operatorname{grad} E$ is the column vector composed of the variational derivatives of $E$ with respect to $X_{1}, X_{2}, X_{3}$ and $\zeta$ (cf. Benjamin \& Olver 1982, equation (A6); Benjamin 1987, equation (2.7)).

The equivalence between (4.10) and the nonlinear conditions (4.2) and (4.3) is readily confirmable. First, by virtue of (4.7), the fourth row of (4.10) recovers (4.2). The first three rows are (with $i=1,2,3$ )

$$
a_{i j} \partial_{t} X_{j}-\gamma_{i} \partial_{t} \zeta=\delta E / \delta X_{i},
$$

that is,

$$
\gamma_{i}\left(\zeta_{(j)} \partial_{t} X_{j}-\partial_{t} \zeta\right)-\zeta_{(i)} \gamma_{j} \partial_{t} X_{j}=\delta E / \delta X_{i} .
$$

Substituting (4.8) for the right-hand side and using (4.2), as already established, to express $\gamma_{j} \partial_{t} X_{j}$ in the third term on the left-hand side, we find that (4.11) reduces to $\gamma_{i}$ times equation (4.3). Hence multiplication by $\gamma_{i}$ and summation proves that (4.3) as well as (4.2) is implied by (4.10) provided $J>0$, which condition can be assumed generally to hold everywhere on $S$.

The generalized Hamiltonian formulation (4.10) is plainly non-canonical. The matrix $\boldsymbol{M}(\boldsymbol{Z})$ is not invertible in any elementary sense, as must be expected because (4.10) covers the whole gauge group of parametric representations of the interface $S$ (a Lie group; cf. footnote to p. 354 in Benjamin 1987). A proof of completeness (closure) for the Hamiltonian structure is given in $\$ 4.4$.

\subsection{Invariants}

The following rule relating certain symmetry groups to invariant functionals has been discussed in a related context by Benjamin (1987, p. 354). Let the four-component 
vector $\boldsymbol{V}$ represent the infinitesimal generator of a one-parameter symmetry group for (4.10), in the sense that if $\boldsymbol{Z}$ is any solution $\boldsymbol{Z}+\varepsilon \boldsymbol{V}$ is also a solution to $O(\varepsilon)$; and let $E$ be unaffected to $O(\varepsilon)$ by the transformation represented. Then a functional $f$ satisfying $\boldsymbol{M}(\boldsymbol{Z}) \boldsymbol{V}=\operatorname{grad} f(\boldsymbol{Z})$ is invariant with $t$ when evaluated on any solution of (4.10).

Because $\boldsymbol{V}=\boldsymbol{Z}_{t}$ represents translation in $t$, which is a symmetry leaving $E$ unaffected if $g=$ const., this rule then shows that $E(\boldsymbol{Z})=$ const. But total-energy conservation is otherwise an immediate consequence of (4.10) if $g=$ const., because we then have

$$
\frac{\mathrm{d} E}{\mathrm{~d} t}=\int_{\Omega}\{\operatorname{grad} E(\boldsymbol{Z})\} \cdot \boldsymbol{Z}_{t} \mathrm{~d} \mu \mathrm{d} v=\int_{\Omega}\left(\boldsymbol{M}(\boldsymbol{Z}) \boldsymbol{Z}_{t}\right) \cdot \boldsymbol{Z}_{t} \mathrm{~d} \mu \mathrm{d} v=0
$$

by the skew-symmetry of $\boldsymbol{M}(\boldsymbol{Z})$.

Generalizing (3.13), expressions for the horizontal impulse invariants are

$$
I_{i}=\int_{\Omega} \zeta \gamma_{i} \mathrm{~d} \mu \mathrm{d} v \quad(i=1,2),
$$

from which there follows

$$
\begin{aligned}
& \operatorname{grad} I_{1}=\left[0, a_{21}, a_{31}, \gamma_{1}\right]^{T}=\boldsymbol{M}(\boldsymbol{Z})[1,0,0,0]^{T}, \\
& \operatorname{grad} I_{2}=\left[a_{12}, 0, a_{32}, \gamma_{2}\right]^{T}=\boldsymbol{M}(\boldsymbol{Z})[0,1,0,0]^{T} .
\end{aligned}
$$

The vector $\boldsymbol{V}=[1,0,0,0]^{T}$ appearing in (4.13) represents the (whole) group of translations in the $x_{1}$-direction, for the addition of any real number $\varepsilon$ to $X_{1}$ shifts the interface by this distance in the $x_{1}$-direction without changing the dynamical equations (4.10). Thus the rule shows that $I_{1}(\boldsymbol{Z})=$ const., as is easily confirmable otherwise. Similarly, $\boldsymbol{V}=[0,1,0,0]^{T}$ appearing in (4.14) represents translations in the $x_{2}$-direction.

Consider next the volume $\mathscr{V}$ displaced upwards by the interface $S$ from the horizontal plane $z=0$, thus

$$
\mathscr{V}=\int_{\Omega} X_{3} \gamma_{3} \mathrm{~d} \mu \mathrm{d} v
$$

Either of the kinematical conditions (4.2) imply that $\mathrm{d} \mathscr{V} / \mathrm{d} t=0$, and plainly the case $\mathscr{V}=0$ is the most typical for the Kelvin-Helmholtz model. The system of equations (4.10) also applies, however, to the case where $\Omega=\mathbb{R} \times \mathbb{R}$ and the model is released from an initial state with non-zero $\mathscr{V}$. It follows from (4.15) that

$$
\operatorname{grad} \mathscr{V}=\left[\gamma_{1}, \gamma_{2}, \gamma_{3}, 0\right]^{T}=\boldsymbol{M}(\boldsymbol{Z})[0,0,0,-1]^{T} .
$$

Thus, according to our rule, the invariance of $\mathscr{V}$ is linked with the obvious symmetry $\zeta-\varepsilon$, which is equivalent to adding $-\varepsilon /\left(\rho-\rho^{\prime}\right)$ to each of $\phi$ and $\phi^{\prime}$. (Note that the terms with factors $U$ and $U^{\prime}$ on the right-hand side of the expression (4.6) for $K$ are unaffected thereby, because the integral of $\gamma_{1}$ over $\Omega$ is zero according to the definition of $\Omega$.)

Further, note that $\boldsymbol{V}=\left[0,0,1,-g\left(\rho-\rho^{\prime}\right) t\right]^{T}$ represents a symmetry group, for it is evident from (4.2) and (4.3) that, if $\left[X_{1}, X_{2}, X_{3}, \zeta\right]^{T}(\mu, v, t)$ is any solution, so also is

$$
\left[X_{1}, X_{2}, X_{3}+\varepsilon, \zeta-g\left(\rho-\rho^{\prime}\right) \varepsilon t\right]^{T},
$$

corresponding to which the transformations of $\phi$ and $\phi^{\prime}$ are

$$
\phi\left(x_{1}, x_{2}, x_{3}-\varepsilon, t\right)-g \varepsilon t, \quad \phi^{\prime}\left(x_{1}, x_{2}, x_{3}-\varepsilon, t\right)-g \varepsilon t .
$$


This symmetry refers to vertical displacements of the whole interface; and in the case $\Omega=\mathbb{R} \times \mathbb{R}$, to keep the Hamiltonian representation meaningful under the transformation (4.17), it is necessary to replace $X_{3}^{2}$ by $\left(X_{3}+\varepsilon\right)^{2}-\varepsilon^{2}$ in the definition (4.4) of potential energy $V$. Thus $E$ is affected to $O(\varepsilon)$ in this case, and so a condition of our rule about symmetries and invariants is unsatisfied. Note, however, that the vertical component of impulse may be defined as

$$
I_{3}=\int_{\Omega} \zeta \gamma_{3} \mathrm{~d} \mu \mathrm{d} \nu
$$

which gives

$$
\operatorname{grad} I_{3}=\left[\begin{array}{llll}
a_{12}, & a_{23}, & 0, & \gamma_{3}
\end{array}\right]^{T}=\boldsymbol{M}(\boldsymbol{Z})[0,0,1,0]^{T} ;
$$

and hence the present symmetry $\mathscr{V}$ appears in

$$
\operatorname{grad}\left(I_{3}+g t\left(\rho-\rho^{\prime}\right) \mathscr{V}\right)=\boldsymbol{M}(\boldsymbol{Z})\left[0,0,1,-g\left(\rho-\rho^{\prime}\right) t\right]^{T} .
$$

This result suggests that $I_{3}+g t\left(\rho-\rho^{\prime}\right)$ may be invariant, and the suggested property may be confirmed as follows. Using (4.20), the skew-symmetry of $\boldsymbol{M}(\boldsymbol{Z})$ and then (4.10), we deduce that

$$
\frac{\mathrm{d} I_{3}}{\mathrm{~d} t}=\int_{\Omega}\left(\operatorname{grad} I_{3}\right) \cdot Z_{t} \mathrm{~d} \mu \mathrm{d} v=-\int_{\Omega} \frac{\delta E}{\delta X_{3}} \mathrm{~d} \mu \mathrm{d} v=-\left[\frac{\partial E}{\partial \varepsilon}\right]_{\varepsilon=0} .
$$

Here $E$ is considered to depend on $\varepsilon$ according to the transformations (4.17) and (4.18). Because plainly $\mathrm{d} K / \mathrm{d} \varepsilon=0$ (when both liquids are infinitely deep), we are left with

$$
\frac{\mathrm{d} I_{3}}{\mathrm{~d} t}=-\left[\frac{\mathrm{d} E}{\mathrm{~d} \varepsilon}\right]_{\varepsilon=0}=-g\left(\rho-\rho^{\prime}\right) \mathscr{V},
$$

which is seen upon comparison with (4.5) and (4.15). (Note that whereas $\mathrm{d} K / \mathrm{d} \varepsilon=0$ is obvious by construction, it may also be confirmed from (4.8) that, equivalently, the integral of $\delta K / \delta X_{3}$ over $\Omega$ is zero. In this integral the coefficients of $\rho$ and $\rho^{\prime}$ are separately found to vanish by virtue of $\phi$ and $\phi^{\prime}$ being harmonic in $\mathscr{D}$ and $\mathscr{D}^{\prime}$ respectively.)

The result (4.21) is special to the case $\Omega=\mathbb{R} \times \mathbb{R}$ with infinitely deep liquids (cf. discussion of $\widehat{I}_{4}$ on p. 171 of Benjamin \& Olver 1982). In this case, if $\mathscr{V}>0$, the upward vertical impulse $I_{3}$ changes at a steady rate equal to minus the relative weight of the liquid in the volume $\mathscr{V}$. In the case of rigid boundaries at finite distances, however, the symmetry now in question is broken, and the extra weight is borne by changes in pressure from its hydrostatic value at the boundaries (cf. Benjamin \& Olver 1982, §6.4).

Finally, let us consider the more complicated symmetry group constituting horizontal Galilean invariance of the hydrodynamic model. The complete group is represented by the statement that if $\left[X_{1}, X_{2}, X_{3}, \zeta\right]^{T}(\mu, v, t)$ is any solution and $\varepsilon$ is any real number, then a new solution is composed by

$$
X_{1}(\mu, v, t)+\varepsilon t, \quad X_{2}(\mu, v, t), \quad X_{3}(\mu, v, t)
$$

and

$$
\zeta(\mu, v, t)-\varepsilon\left(\rho-\rho^{\prime}\right) X_{1}(\mu, v, t)-\left(\rho U-\rho^{\prime} U^{\prime}\right) \varepsilon t+\frac{1}{2}\left(\rho-\rho^{\prime}\right) \varepsilon^{2} t,
$$


corresponding to which the transformed velocity potentials are

$$
\left.\begin{array}{r}
\phi\left(x_{1}-\varepsilon t, x_{2}, x_{3}, t\right)+\varepsilon\left(x_{1}-U t\right)+\frac{1}{2} \varepsilon^{2} t, \\
\phi^{\prime}\left(x_{1}-\varepsilon t, x_{2}, x_{3}, t\right)+\varepsilon\left(x_{1}-U^{\prime} t\right)+\frac{1}{2} \varepsilon^{2} t .
\end{array}\right\}
$$

This statement is easily verified by substitution of (4.22)-(4.24) into (4.2) and (4.3).

The infinitesimal generator of the symmetry group is accordingly represented by

$$
\boldsymbol{V}=\left[t, 0,0,\left(\rho-\rho^{\prime}\right) X_{1}-\left(\rho U-\rho^{\prime} U^{\prime}\right) t\right]^{T} .
$$

In relation to Hamiltonian structure and a conservation law, however, this symmetry requires more careful appraisal than the previous ones because $K$ defined by (4.6) does not remain finite under the transformations (4.24). Note first that, in place of (4.22)-(4.24), a transformation composing a new solution is just $X_{1}+\varepsilon t$, with $X_{2}, X_{3}$ and $\zeta$ unchanged, while $U$ and $U^{\prime}$ become $U+\varepsilon$ and $U^{\prime}+\varepsilon$. We then have merely $\boldsymbol{V}=[t, 0,0,0]^{T}$, which appears in $\operatorname{grad}\left(I_{1} t\right)=\boldsymbol{M}(\boldsymbol{Z})[t, 0,0,0]^{T}$, but $E$ depends on $\varepsilon$. The symmetry is hence seen to imply the trivial identity $\mathrm{d}\left(I_{1} t\right) / \mathrm{d} t=I_{1}=(\partial E / \partial \varepsilon)_{\varepsilon=0}$ (cf. Benjamin 1987, equation (2.10)).

A more interesting result is found by considering

$$
F=\int_{\Omega} X_{1} X_{3} \gamma_{3} \mathrm{~d} \mu \mathrm{d} v,
$$

which in the case $\mathscr{V}>0$ is equivalent to $\mathscr{V} \bar{X}_{1}$, where $\bar{X}_{1}$ is the horizontal coordinate of the centroid of the displaced volume. It follows from (4.26) that

$$
\operatorname{grad} F=\left[X_{1} \gamma_{1}, X_{1} \gamma_{2}, X_{1} \gamma_{3}, 0\right]^{T}=\boldsymbol{M}(\boldsymbol{Z})\left[0,0,0,-X_{1}\right]^{T} .
$$

Hence, also in view of (4.13) and (4.16), we have

$$
\operatorname{grad}\left\{I_{1} t-\left(\rho-\rho^{\prime}\right) F+\left(\rho U-\rho^{\prime} U^{\prime}\right) \mathscr{V} t\right\}=\boldsymbol{M}(\boldsymbol{Z}) \boldsymbol{V}
$$

with $\boldsymbol{V}$ given by (4.25).

The functional on the left-hand side of (4.27) is thus indicated as a possible invariant. Because $I_{1}$ and $\mathscr{V}$ are already known to be invariant, the indication is that

$$
\left(\rho-\rho^{\prime}\right) \frac{\mathrm{d} F}{\mathrm{~d} t}=I_{1}+\left(\rho U-\rho^{\prime} U^{\prime}\right) \mathscr{V} ;
$$

and this property can be checked directly. Using the first of (4.2), we obtain

$$
\begin{aligned}
\frac{\mathrm{d} F}{\mathrm{~d} t} & =\int_{\Omega}(\operatorname{grad} F) \cdot Z_{t} \mathrm{~d} \mu \mathrm{d} v=\int_{\Omega} X_{1} \gamma_{i}\left(X_{i}\right)_{t} \mathrm{~d} \mu \mathrm{d} v \\
& =\int_{\Omega} X_{1}\left(U \gamma_{1}+\Phi_{(n)} J\right) \mathrm{d} \mu \mathrm{d} v+U \mathscr{V}+\int_{\Omega} X_{1} \Phi_{(n)} J \mathrm{~d} \mu \mathrm{d} v ;
\end{aligned}
$$

and the second of (4.2) leads to a similar result with $U^{\prime}$ replacing $U$ and $\Phi_{(n)}^{\prime}$ replacing $\Phi_{(n)}$. Hence (4.28) is confirmed if

$$
\int_{\Omega} X_{1}\left(\rho \Phi_{(n)}-\rho^{\prime} \Phi_{(n)}^{\prime}\right) J \mathrm{~d} \mu \mathrm{d} v=I_{1} .
$$

This identity is an immediate consequence of Green's theorem in the case of motions such that $\phi$ and $\phi^{\prime}$ are periodic in $x_{1}$ and $x_{2}$. In the case of localized threedimensional motions, Green's theorem may be referred to the harmonic functions $\phi$ and $x_{1}=r \cos \theta$ in the space $\mathscr{D}$ bounded by the interface $S$ and, below it, a hemisphere 
of radius $r \rightarrow \infty$, on which spherical polar coordinates $\theta$ and $\psi$ range over $[-\pi, 0]$ and $[0, \pi]$ respectively. Thus, because $\left(\partial x_{1} / \partial n\right) J=\gamma_{1}$ on $S$, the theorem shows that

$$
\begin{aligned}
\int_{\Omega}\left(X_{1} \Phi_{(n)} J-\Phi \gamma_{1}\right) \mathrm{d} \mu \mathrm{d} v & =\lim _{r \rightarrow \infty} \int\left[r \frac{\partial \phi}{\partial r}-\phi\right] \cos \theta r^{2} \sin \theta \mathrm{d} \theta \mathrm{d} \psi \\
& =-2 \pi C,
\end{aligned}
$$

where $C$ is the coefficient of the dipole far field aligned with the $x_{1}$-axis, as was defined in the discussion of (3.2). A corresponding conclusion holds for the space above $S$, in which the dipole coefficient of the far field is $C^{\prime}$. Hence a generalized version of (4.29) is inferred, wherein a term $-2 \pi\left(\rho C+\rho^{\prime} C^{\prime}\right)=-4 \pi \rho C$ is added to the right-hand side. In general the same term appears on the right-hand side of (4.28); and so the unmodified conservation law (4.28) depends on the assumption $C=C^{\prime}=0$ that was introduced with explanation in the discussion of (3.2).

This conservation law is comparable with a result demonstrated for water waves by Benjamin \& Olver (1982, equation (6.24)). Its version with the extra term due to the dipole far fields is comparable with a result demonstrated for bubbles by Benjamin (1987, equation (2.18); note that for bubbles the definition of impulse $I_{1}$ has a sign opposite to the present definition (4.12) of $I_{1}$ ).

\subsection{Action and the symplectic operator}

A technical requirement for completeness of the specification of the Hamiltonian structure in $\S 4.2$ is that the skew-symmetric operator $\boldsymbol{M}(\boldsymbol{Z})$ be closed. The skewsymmetric bilinear form associated with $\boldsymbol{M}(\boldsymbol{Z})$ is

$$
\omega\left(\boldsymbol{\xi}_{1}, \boldsymbol{\xi}_{2}\right)=\int_{\Omega}\left\langle\boldsymbol{\xi}_{1}, \boldsymbol{M}(\boldsymbol{Z}) \boldsymbol{\xi}_{2}\right\rangle \mathrm{d} \mu \mathrm{d} v
$$

where $\langle\cdot, \cdot\rangle$ is a standard inner product for vector-valued functions of the form (4.9) and $\xi_{1}$ and $\xi_{2}$ are arbitrary vectors of the form (4.9).

The differential two-form associated with $\omega$ is

$$
\begin{aligned}
\omega=\int_{\Omega}\{ & a_{12} \mathrm{~d} X_{1} \wedge \mathrm{d} X_{2}+a_{13} \mathrm{~d} X_{1} \wedge \mathrm{d} X_{3}-\gamma_{1} \mathrm{~d} X_{1} \wedge \mathrm{d} \zeta \\
& \left.+a_{23} \mathrm{~d} X_{2} \wedge \mathrm{d} X_{3}-\gamma_{2} \mathrm{~d} X_{2} \wedge \mathrm{d} \zeta-\gamma_{3} \mathrm{~d} X_{3} \wedge \mathrm{d} \zeta\right\} \mathrm{d} \mu \mathrm{d} v
\end{aligned}
$$

where $\wedge$ is the wedge product. We say that the skew-symmetric operator $\boldsymbol{M}(\boldsymbol{Z})$ satisfies the closure condition if $\mathrm{d} \omega=0$ where $\mathrm{d}$ is the exterior derivative (cf. Benjamin 1987, Appendix B; Bowman 1987 and references therein). The direct analysis of $\mathrm{d} \omega$ to verify closure is lengthy and not very illuminating. Here we will circumvent this difficulty by showing that $\omega$ is an exact two-form; that is there exists a one-form $\theta$ with $\omega=\mathrm{d} \theta$. It then follows immediately that $\mathrm{d} \omega=\mathrm{d}^{2} \theta=0$. An interesting consequence of this geometric proof of closure is that the one-form $\theta$ corresponds to wave action (cf. Hayes 1970). Indeed the result gives a generalization of wave action to overhanging and parametrically defined interfacial waves.

The wave-action functional, for the case when the surface is defined parametrically, is found to correspond to the integral over the fluid interface of the product of the weighted potential $\zeta$ and the normal component of the time-derivative of the interface position, $\boldsymbol{X}_{t} \cdot \boldsymbol{n}$,

$\mathscr{A}(\boldsymbol{Z})=\int_{S} \zeta \boldsymbol{X}_{t} \cdot \boldsymbol{n} \mathrm{d} S=\int_{\Omega} \zeta \boldsymbol{X}_{t} \cdot \boldsymbol{n} J \mathrm{~d} \mu \mathrm{d} v=\int_{\Omega} \zeta\left(\gamma_{1} \frac{\partial X_{1}}{\partial t}+\gamma_{2} \frac{\partial X_{2}}{\partial t}+\gamma_{3} \frac{\partial X_{3}}{\partial t}\right) \mathrm{d} \mu \mathrm{d} v$. 
The one-form corresponding to action is then

$$
\theta=\int_{\Omega} \zeta\left(\gamma_{1} \mathrm{~d} X_{1}+\gamma_{2} \mathrm{~d} X_{2}+\gamma_{3} \mathrm{~d} X_{3}\right) \mathrm{d} \mu \mathrm{d} \nu .
$$

It is now verified that $\mathrm{d} \theta=\omega$.

By direct calculation, using the properties of exterior differentiation,

$$
\begin{aligned}
\mathrm{d} \theta= & \int_{\Omega}\left\{\gamma_{1} \mathrm{~d} \zeta \wedge \mathrm{d} X_{1}+\gamma_{2} \mathrm{~d} \zeta \wedge \mathrm{d} X_{2}+\gamma_{3} \mathrm{~d} \zeta \wedge \mathrm{d} X_{3}\right. \\
& \left.+\zeta \mathrm{d} \gamma_{1} \wedge \mathrm{d} X_{1}+\zeta \mathrm{d} \gamma_{2} \wedge \mathrm{d} X_{2}+\zeta \mathrm{d} \gamma_{3} \wedge \mathrm{d} X_{3}\right\} \mathrm{d} \mu \mathrm{d} v .
\end{aligned}
$$

But, using the definition of $\gamma_{j}(j=1,2,3)$ in $\S 4.1$,

$$
\begin{aligned}
& \mathrm{d} \gamma_{1}=X_{3 v} \mathrm{~d} X_{2 \mu}+X_{2 \mu} \mathrm{d} X_{3 v}-X_{3 \mu} \mathrm{d} X_{2 v}-X_{2 v} \mathrm{~d} X_{3 \mu}, \\
& \mathrm{d} \gamma_{2}=X_{1 v} \mathrm{~d} X_{3 \mu}+X_{3 \mu} \mathrm{d} X_{1 v}-X_{1 \mu} \mathrm{d} X_{3 v}-X_{3 v} \mathrm{~d} X_{1 \mu}, \\
& \mathrm{d} \gamma_{3}=X_{1 \mu} \mathrm{d} X_{2 v}+X_{2 v} \mathrm{~d} X_{1 \mu}-X_{1 v} \mathrm{~d} X_{2 \mu}-X_{2 \mu} \mathrm{d} X_{1 v},
\end{aligned}
$$

from which it follows, after a short calculation, that

$$
\begin{aligned}
\sum_{j=1}^{3} \mathrm{~d} \gamma_{j} \wedge \mathrm{d} X_{j} & =\frac{\partial}{\partial \mu}\left(-X_{3 v} \mathrm{~d} X_{1} \wedge \mathrm{d} X_{2}+X_{2 v} \mathrm{~d} X_{1} \wedge \mathrm{d} X_{3}-X_{1 v} \mathrm{~d} X_{2} \wedge \mathrm{d} X_{3}\right) \\
& +\frac{\partial}{\partial v}\left(X_{3 \mu} \mathrm{d} X_{1} \wedge \mathrm{d} X_{2}-X_{2 \mu} \mathrm{d} X_{1} \wedge \mathrm{d} X_{3}+X_{1 \mu} \mathrm{d} X_{2} \wedge \mathrm{d} X_{3}\right)
\end{aligned}
$$

To simplify $\mathrm{d} \theta$ further note that

$$
\zeta_{\mu}=\zeta_{(1)} \frac{\partial X_{1}}{\partial \mu}+\zeta_{(2)} \frac{\partial X_{2}}{\partial \mu}+\zeta_{(3)} \frac{\partial X_{3}}{\partial \mu}, \quad \zeta_{v}=\zeta_{(1)} \frac{\partial X_{1}}{\partial v}+\zeta_{(2)} \frac{\partial X_{2}}{\partial v}+\zeta_{(3)} \frac{\partial X_{3}}{\partial v},
$$

and therefore the coefficients in $\boldsymbol{M}(\boldsymbol{Z})$ can be written in the form

$$
a_{12}=\zeta_{\mu} \frac{\partial X_{3}}{\partial v}-\zeta_{v} \frac{\partial X_{3}}{\partial \mu}, \quad a_{13}=\zeta_{v} \frac{\partial X_{2}}{\partial \mu}-\zeta_{\mu} \frac{\partial X_{2}}{\partial v}, \quad a_{23}=\zeta_{\mu} \frac{\partial X_{1}}{\partial v}-\zeta_{v} \frac{\partial X_{1}}{\partial \mu} .
$$

Combining the above results in the expression for $\mathrm{d} \theta$ gives

$$
\begin{aligned}
& \mathrm{d} \theta= \int_{\Omega}\left\{\gamma_{1} \mathrm{~d} \zeta \wedge \mathrm{d} X_{1}+\gamma_{2} \mathrm{~d} \zeta \wedge \mathrm{d} X_{2}+\gamma_{3} \mathrm{~d} \zeta \wedge \mathrm{d} X_{3}\right. \\
&+\zeta \frac{\partial}{\partial \mu}\left(-X_{3 v} \mathrm{~d} X_{1} \wedge \mathrm{d} X_{2}+X_{2 v} \mathrm{~d} X_{1} \wedge \mathrm{d} X_{3}-X_{1 v} \mathrm{~d} X_{2} \wedge \mathrm{d} X_{3}\right) \\
&\left.+\zeta \frac{\partial}{\partial v}\left(X_{3 \mu} \mathrm{d} X_{1} \wedge \mathrm{d} X_{2}-X_{2 \mu} \mathrm{d} X_{1} \wedge \mathrm{d} X_{3}+X_{1 \mu} \mathrm{d} X_{2} \wedge \mathrm{d} X_{3}\right)\right\} \mathrm{d} \mu \mathrm{d} v \\
&= \int_{\Omega}\left\{-\gamma_{1} \mathrm{~d} X_{1} \wedge \mathrm{d} \zeta-\gamma_{2} \mathrm{~d} X_{2} \wedge \mathrm{d} \zeta-\gamma_{3} \mathrm{~d} X_{3} \wedge \mathrm{d} \zeta\right. \\
& \quad-\zeta_{\mu}\left(-X_{3 v} \mathrm{~d} X_{1} \wedge \mathrm{d} X_{2}+X_{2 v} \mathrm{~d} X_{1} \wedge \mathrm{d} X_{3}-X_{1 v} \mathrm{~d} X_{2} \wedge \mathrm{d} X_{3}\right) \\
&\left.\quad-\zeta_{v}\left(X_{3 \mu} \mathrm{d} X_{1} \wedge \mathrm{d} X_{2}-X_{2 \mu} \mathrm{d} X_{1} \wedge \mathrm{d} X_{3}+X_{1 \mu} \mathrm{d} X_{2} \wedge \mathrm{d} X_{3}\right)\right\} \mathrm{d} \mu \mathrm{d} v \\
&=\int_{\Omega}\left\{-\gamma_{1} \mathrm{~d} X_{1} \wedge \mathrm{d} \zeta-\gamma_{2} \mathrm{~d} X_{2} \wedge \mathrm{d} \zeta-\gamma_{3} \mathrm{~d} X_{3} \wedge \mathrm{d} \zeta\right. \\
&=\omega . \quad
\end{aligned}
$$

In the above argument we have used the anti-symmetry of the wedge, integration by 
parts (assuming suitable conditions on variations at the boundary of the region $\Omega$ ), the expressions for $a_{i j}$ and finally the definition of $\omega$.

The action may be used to construct a variational principle for (4.10). Taking suitable variations with fixed endpoint conditions at $t_{1}, t_{2}$ it follows that

$$
\operatorname{grad} \mathscr{A}(\boldsymbol{Z})=\boldsymbol{M}(\boldsymbol{Z}) \boldsymbol{Z}_{t}
$$

where the gradient is defined with respect to an inner product that includes integration over $\mu, v$ and $t$. Therefore, for all intervals $\left[t_{1}, t_{2}\right] \subset \mathbb{R}$ and admissable variations with fixed endpoint conditions at $\left(t_{1}, t_{2}\right)$, the Hamiltonian evolution equation (4.10) is given by the vanishing of the first variation of the functional,

$$
L\left(\boldsymbol{Z}, \boldsymbol{Z}_{t}\right)=\int_{t_{1}}^{t_{2}}[\mathscr{A}(\boldsymbol{Z})-E(\boldsymbol{Z})] \mathrm{d} t .
$$

In the special case where the interface can be represented as a single-valued function of position, say $X_{3}=\eta\left(x_{1}, x_{2}\right)$ and so $X_{t}=\left(0,0, \eta_{t}\right)$, the action reduces to

$$
\mathscr{A}=\int_{\Omega} \zeta \eta_{t} \mathrm{~d} x_{1} \mathrm{~d} x_{2}
$$

When $\rho=1$ and $\rho^{\prime}=0$ the expression for action reduces to $\mathscr{A}=\int_{\Omega} \Phi \eta_{t} \mathrm{~d} x_{1} \mathrm{~d} x_{2}$ which recovers the classical result for water waves (cf. Benjamin \& Olver 1982, p. 168).

\subsection{Two-dimensional motions}

Problems in this class are covered by letting $v=x_{2}=X_{2}$ and taking as three dependent variables

$$
X_{1}(\mu, t), \quad X_{3}(\mu, t), \quad \zeta(\mu, t)
$$

(so that $\gamma_{2}=0$ ). Again using $\boldsymbol{Z}$ to denote them collectively as a column vector, and writing $\operatorname{grad} E$ for the column vector composed of the three variational derivatives, we have the same Hamiltonian representation (4.10) but with

$$
\boldsymbol{M}(\boldsymbol{Z})=\left[\begin{array}{ccc}
0 & a_{13} & -\gamma_{1} \\
a_{31} & 0 & -\gamma_{3} \\
\gamma_{1} & \gamma_{3} & 0
\end{array}\right],
$$

in which $\gamma_{1}=-\partial X_{3} / \partial \mu, \gamma_{3}=\partial X_{1} / \partial \mu$ and

$$
a_{13}=-\zeta_{(1)} \frac{\partial X_{1}}{\partial \mu}-\zeta_{(3)} \frac{\partial X_{3}}{\partial \mu}=-\zeta_{\mu} .
$$

Horizontal impulse is expressed by

$$
I_{1}=-\int_{\Omega} \zeta \frac{\partial X_{3}}{\partial \mu} \mathrm{d} \mu
$$

Here, as in the integrals expressing $E$, the range $\Omega$ of integration is either $\mathbb{R}$, in the case of localized motions, or one period in the case of spatially periodic motions. From (4.32) and (4.31), the property (4.13) is seen to be recovered in the form

$$
\operatorname{grad} I_{1}=\left[0, \partial \zeta / \partial \mu,-\partial X_{3} / \partial \mu\right]^{T}=\boldsymbol{M}(\boldsymbol{Z})[1,0,0]^{T},
$$

which reconfirms that $I_{1}=$ const. along any orbit of the Hamiltonian system. For a steady wave motion, we have

$$
X_{1}=X_{1}(\mu)+c t, \quad X_{3}=X_{3}(\mu), \quad \zeta=\zeta(\mu)
$$


and thus (4.32) obviously bears out the variational characterization (3.14); that is,

$$
\boldsymbol{M}(\boldsymbol{Z})\left(\partial_{t} \boldsymbol{Z}\right)=c \operatorname{grad} I_{1} .
$$

Note that, in the case of periodic motions, the period in $\mu$ can generally be chosen to be the same as the period in $x_{1}$, say $\lambda$. Then

$$
X_{1}(\mu+n \lambda, t)=X_{1}(\mu, t)+n \lambda, \quad X_{3}(\mu+n \lambda, t)=X_{3}(\mu, t), \quad \zeta(\mu+n \lambda, t)=\zeta(\mu, t),
$$

where $n$ is any integer.

The action functional for two-dimensional motions restricts to

$$
\mathscr{A}(\boldsymbol{Z})=\int_{\Omega} \zeta\left(\gamma_{1} \frac{\partial X_{1}}{\partial t}+\gamma_{3} \frac{\partial X_{3}}{\partial t}\right) \mathrm{d} \mu=\int_{\Omega} \zeta\left(\frac{\partial X_{1}}{\partial \mu} \frac{\partial X_{3}}{\partial t}-\frac{\partial X_{3}}{\partial \mu} \frac{\partial X_{1}}{\partial t}\right) \mathrm{d} \mu
$$

and, as in the three-dimensional case, $\operatorname{grad} \mathscr{A}(\boldsymbol{Z})=\boldsymbol{M}(\boldsymbol{Z}) \boldsymbol{Z}_{t}$. But

$$
\boldsymbol{M}(\boldsymbol{Z}) \boldsymbol{Z}_{t}=\left(\begin{array}{c}
\zeta_{t} X_{3 \mu}-\zeta_{\mu} X_{3 t} \\
-\zeta_{t} X_{1 \mu}+\zeta_{\mu} X_{1 t} \\
-X_{1 t} X_{3 \mu}+X_{1 \mu} X_{3 t}
\end{array}\right)=\boldsymbol{Z}_{\mu} \times \boldsymbol{Z}_{t}
$$

where $x$ is the usual vector cross-product in $\mathbb{R}^{3}$ (noting that $\boldsymbol{Z}$ has only three components in the two-dimensional case). Therefore the Hamiltonian equations (4.10) reduce to the elegant form

$$
\boldsymbol{Z}_{\mu} \times \boldsymbol{Z}_{t}=\operatorname{grad} E(\boldsymbol{Z}) .
$$

\section{Comments on the linearized approximation}

Returning to the linearized theory summarized in $\$ 2.1$, we now note its Hamiltonian formulation which provides an informative interpretation of Kelvin-Helmholtz instability. In this approximation, the Hamiltonian representation (3.11) of the evolutionary problem remains valid, of course, but $E$ reduces to a quadratic functional of the dependent variables $\eta$ and $\zeta$. An explicit expression for $E$ can be deduced as follows.

First note the admissible simplification of the functional $\mathscr{K}$ given by (3.3), the minimum of which according to (3.4) defines the kinetic-energy perturbation $K$ as a functional of $\eta$ and $\zeta$ alone. The domains of integration $\mathscr{D}$ and $\mathscr{D}^{\prime}$ in (3.3), which are defined exactly by (3.1), can be approximated by $\Omega \times(-\infty, 0)$ and $\Omega \times(0, \infty)$ without affecting $K$ to second order. Hence the minimizer $\left(\phi, \phi^{\prime}\right)$ of $\mathscr{K}$ subject to the constraint specified in (3.4) which makes $\rho \Phi-\rho^{\prime} \Phi^{\prime}=\zeta$, where now $\Phi=\phi(x, y, 0, t)$ and $\Phi^{\prime}=\phi^{\prime}(x, y, 0, t)$, is easily confirmed to have the property

$$
\Phi_{(z)}-U \eta_{x}=\Phi_{(z)}^{\prime}-U^{\prime} \eta_{x}
$$

with $\Phi_{(z)}=\phi_{z}(x, y, 0, t)$ and $\Phi_{(z)}^{\prime}=\phi_{z}^{\prime}(x, y, 0, t)$. This property is just the linearized version of (3.6). Moreover, we can write $\Phi_{(z)}=\mathrm{L} \Phi, \Phi_{(z)}^{\prime}=-\mathrm{L} \Phi^{\prime}$, where $\mathrm{L}$ is the linear operator whose symbol is $k$ or more generally $|k|$. (The symbol $\widehat{\mathrm{L}}$ of a linear operator on functions of $(x, y)$ is defined as the multiplier in the relation $\mathrm{Le}^{\mathrm{i}(\alpha x+\beta y)}=\widehat{\mathrm{L}}(\alpha, \beta) \mathrm{e}^{\mathrm{i}(\alpha x+\beta y)}$. The present identification $\widehat{\mathrm{L}}=k=\left(\alpha^{2}+\beta^{2}\right)^{1 / 2}$, with $k>0$ understood, is evident from the periodic harmonic functions $\phi$ and $\phi^{\prime}$ exhibited in §2.1. Note that Green's theorem for harmonic functions shows the present $\mathrm{L}$ to be a symmetric operator, which fact also appears from Parseval's theorem referred to a Fourier-integral representation of $\mathrm{L} f(x, y) \in \mathrm{L}_{2}(\mathbb{R} \times \mathbb{R})$.) 
Solving (5.1) and the definition $\rho \Phi-\rho^{\prime} \Phi^{\prime}=\zeta$ as simultaneous equations for $\Phi$ and $\Phi^{\prime}$, we find that

$$
\begin{aligned}
\Phi & =\frac{1}{\rho+\rho^{\prime}}\left[\zeta+\rho^{\prime}\left(U-U^{\prime}\right) \mathrm{L}^{-1} \eta_{x}\right], \\
\Phi^{\prime} & =\frac{1}{\rho+\rho^{\prime}}\left[-\zeta+\rho\left(U-U^{\prime}\right) \mathrm{L}^{-1} \eta_{x}\right] .
\end{aligned}
$$

Hence the second-order approximation to $E=K+V$ is found to have the form

$$
E=\int_{\Omega}\left[\frac{1}{2} \zeta \mathrm{A} \zeta-\zeta \mathrm{B} \eta+\frac{1}{2} \eta \mathrm{C} \eta\right] \mathrm{d} x \mathrm{~d} y
$$

in which

$$
\begin{aligned}
& \mathrm{A} \zeta=\frac{1}{\rho+\rho^{\prime}} \mathrm{L} \zeta, \quad \mathrm{B} \eta=\frac{\rho U+\rho^{\prime} U^{\prime}}{\rho+\rho^{\prime}} \eta_{x}, \\
& \mathrm{C} \eta=\frac{\rho \rho^{\prime}\left(U-U^{\prime}\right)^{2}}{\rho+\rho^{\prime}} \mathrm{L}^{-1} \eta_{x x}+g\left(\rho-\rho^{\prime}\right) \eta-\sigma\left(\eta_{x x}+\eta_{y y}\right) .
\end{aligned}
$$

Here the second-order approximation $R-1=\frac{1}{2}\left(\eta_{x}^{2}+\eta_{y}^{2}\right)$ has been used for part of $V$ given by (3.9). The linear operators $\mathrm{A}$ and $\mathrm{C}$ thus defined are evidently symmetric, and $\mathrm{B}$ is anti-symmetric. The symbols of these operators are

$$
\left.\begin{array}{l}
\widehat{\mathrm{A}}=\frac{k}{\rho+\rho^{\prime}}, \quad \widehat{\mathrm{B}}=\frac{\rho U+\rho^{\prime} U^{\prime}}{\rho+\rho^{\prime}} \mathrm{i} \alpha, \\
\widehat{\mathrm{C}}=\frac{\rho \rho^{\prime}\left(U-U^{\prime}\right)^{2}}{\rho+\rho^{\prime}}\left(-\frac{\alpha^{2}}{k}\right)+g\left(\rho-\rho^{\prime}\right)+\sigma k^{2} .
\end{array}\right\}
$$

In view of the Hamiltonian representation (3.11), the linearized equations of motion are seen at once from (5.2) to be

$$
\eta_{t}=\mathrm{A} \zeta-\mathrm{B} \eta, \quad \zeta_{t}-\mathrm{B} \zeta-\mathrm{C} \eta,
$$

which may be confirmed to correspond to (2.3) and (2.4). Focusing attention again on solutions that are simple-harmonic in $x$ and $y$, so writing

$$
\eta=\operatorname{Re}\left(\widehat{\eta} \mathrm{e}^{s t} \exp \{\mathrm{i}(\alpha x+\beta y)\}\right) \quad \text { and } \zeta=\operatorname{Re}\left(\widehat{\zeta} \mathrm{e}^{s t} \exp \{\mathrm{i}(\alpha x+\beta y)\}\right)
$$

we obtain from (5.4)

$$
(\widehat{\mathrm{B}}+s) \widehat{\eta}-\widehat{\mathrm{A}} \widehat{\zeta}=0, \quad \widehat{\mathrm{C}} \widehat{\eta}+(\widehat{\mathrm{B}}+s) \widehat{\zeta}=0
$$

which shows that

$$
s=-\widehat{\mathrm{B}} \pm(-\widehat{\mathrm{A}} \widehat{\mathrm{C}})^{1 / 2} .
$$

Substitution of the expressions (5.3) into this result recovers the classic formula (2.6) exactly.

Let us now evaluate $E$ for such solutions of (5.4). Assuming $\beta \neq 0$, and taking $\Omega$ to be composed of one period $2 \pi /|\alpha|$ in $x$ and one period $2 \pi /|\beta|$ in $y$, we see that the sum of the first and third terms on the right-hand side of (5.2) is

$$
\frac{\pi^{2}}{|\alpha \beta|}\left(\widehat{\mathrm{A}}|\widehat{\zeta}|^{2}+\widehat{\mathrm{C}}|\widehat{\eta}|^{2}\right) \mathrm{e}^{2 t \operatorname{Re}(s)}=\frac{\pi^{2}}{|\alpha \beta|}(|\widehat{\mathrm{C}}|+\widehat{\mathrm{C}})|\widehat{\eta}|^{2} \mathrm{e}^{2 t \operatorname{Re}(s)},
$$

because $\widehat{\mathrm{A}}>0$ while (5.5) and (5.6) imply that $\widehat{\zeta}= \pm(-\widehat{\mathrm{C}} / \widehat{\mathrm{A}})^{1 / 2} \widehat{\eta}$. If $\beta=0$ and $\Omega$ is 
just one period $2 \pi /|\alpha|$ in $x$, the factor $\pi^{2} /|\alpha \beta|$ in (5.7) is replaced by $\pi / 2|\alpha|$. In the case $\widehat{\mathrm{C}}>0, \zeta$ is in phase with $\mathrm{B} \eta$ or $-\mathrm{B} \eta$, and then the second term on the right-hand side of (5.2) is

$$
\pm\left(\frac{2 \pi^{2}}{|\alpha \beta|}\right)|\widehat{\mathrm{B}}|(\widehat{\mathrm{C}} / \widehat{\mathrm{A}})^{1 / 2}|\widehat{\eta}|^{2}
$$

or the same with factor $\pi /|\alpha|$ if $\beta=0$. (Note that $\operatorname{Re}(s)=0$ in this case.) But, in the case $\widehat{\mathrm{C}}<0, \zeta$ is in phase with $\eta$ or $-\eta$, hence in quadrature with $\mathrm{B} \eta$, and so the second term on the right-hand side of (5.2) is zero.

In view also of (5.7), we conclude that $E=0$ for the non-trivial solutions of (5.4) when $\widehat{\mathrm{C}}<0$, one of which solutions has the exponential growth rate $\operatorname{Re} s=$ $(-\widehat{\mathrm{A}} \widehat{\mathrm{C}})^{1 / 2}>0$ constituting Kelvin-Helmholtz instability. When $\widehat{\mathrm{C}}>0$, on the other hand, we have $\operatorname{Re} s=0$ and $E$ has the constant values

$$
\frac{2 \pi^{2}}{|\alpha \beta|}\left\{\widehat{\mathrm{C}} \pm|\widehat{\mathrm{B}}|(\widehat{\mathrm{C}} / \widehat{\mathrm{A}})^{1 / 2}\right\}|\widehat{\eta}|^{2},
$$

one of which is positive for all $\widehat{\mathrm{C}}>0$ but the other is negative for sufficiently small $\widehat{\mathrm{C}}>0$.

Thus a property common to all Hamiltonian systems is explicitly demonstrated. Namely, when a parameter characterizing a steady state of the system is varied continuously through a critical value, on one side of which infinitesimal perturbed motions are time-periodic and on the other side of which the steady state becomes unstable, then the limit of stability corresponds to the coincidence of time-periodic modes for which respectively $E>0$ and $E<0$ in a neighbourhood of the critical value on the stable side. In the modern literature the term 'different Krein signatures' is often used to describe $E>0$ and $E<0$; but the general property in question was recognized long ago by Weierstrass and Kelvin.

The critical case $\widehat{\mathrm{C}}=0$ also provides instability, albeit in weaker form than the exponentially growing solutions of (5.4) provided when $\widehat{\mathrm{C}}<0$. In this case (5.4) is satisfied by

$$
(\eta, \zeta)=\frac{1}{2}(t \widehat{\mathrm{A}} \widehat{\zeta}, \widehat{\zeta}) \exp \{-\widehat{\mathrm{B}} t+\mathrm{i}(\alpha x+\beta y)\}+\text { c.c. }
$$

for any complex constant $\widehat{\zeta}$; and this solution will negate Liapunov stability in any appropriate metric.

It has been recognized in $\S 2.1$ that, when $\left(U-U^{\prime}\right)^{2}$ is large enough to satisfy the inequality (2.6), Kelvin-Helmholtz instability arises over a range of $\alpha>0$ and $\beta \geqslant 0$. Values of $\left(U-U^{\prime}\right)^{2}$ are suitably represented in the dimensionless form

$$
F=\frac{\rho \rho^{\prime}\left(U-U^{\prime}\right)^{2}}{\left(\rho+\rho^{\prime}\right)\left[g\left(\rho-\rho^{\prime}\right) \sigma\right]^{1 / 2}} ;
$$

and the critical values of $F$ making $\widehat{\mathrm{C}}=0$ are given by

$$
F_{c}=\frac{\left(\bar{\alpha}^{2}+\bar{\beta}^{2}\right)^{1 / 2}\left(1+\bar{\alpha}^{2}+\bar{\beta}^{2}\right)}{\bar{\alpha}^{2}},
$$

with $\bar{\alpha}=\alpha\left[\sigma / g\left(\rho-\rho^{\prime}\right)\right]^{1 / 2}$ and $\bar{\beta}=\beta\left[\sigma / g\left(\rho-\rho^{\prime}\right)\right]^{1 / 2}$. The possibilities are illustrated in figure 2 , which shows graphs of $F_{c}$ against $\bar{\alpha}$ for several values of $\bar{\beta}$. At points $(F, \bar{\alpha})$ lying above the curve for a particular $\bar{\beta} \geqslant 0$, the system (5.4) has a solution growing exponentially in time with the respective wavenumbers $\bar{\alpha}$ and $\bar{\beta}$. As already 


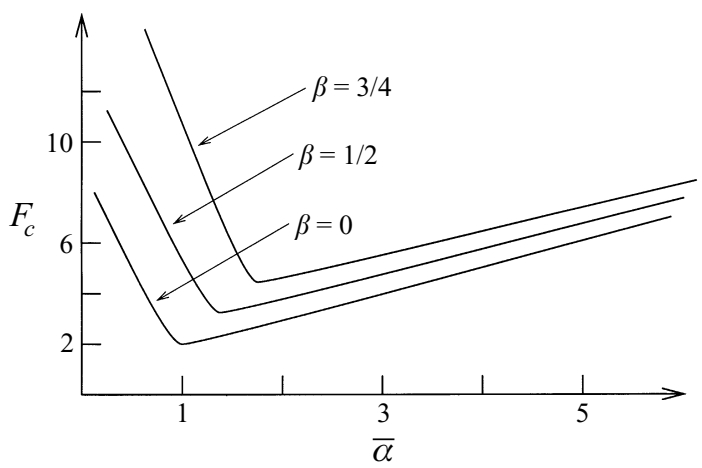

FIGURE 2. Schematic diagram showing the dimensionless neutral curve $F_{c}$ plotted against non-dimensional wavenumber $\bar{\alpha}$ for several values of $\bar{\beta}$. Kelvin-Helmholtz instability arises for $F>F_{c}(\bar{\alpha}, \bar{\beta})$.

pointed out in $\S 2.1$, the unstable range of $\bar{\alpha}$ when $F>2$ is widest for $\beta=0$; and the minimum value of $F$ for instability is $F_{c}=2$ which is achieved at $\bar{\alpha}=1, \bar{\beta}=0$, which suggests that a form of Squire's Theorem may be operational.

\section{Conclusions}

The main contribution of this paper has been to identify the Hamiltonian structure of the Kelvin-Helmholtz problem. This structure is far from obvious; it depends crucially on the variational characterization of the kinetic-energy perturbation introduced in $\$ 3.1$; and it has not been noticed before. Its advantages include the systematic relation of symmetries to conservation laws, several of which demonstrated in $\$ 3.3$ and $\S 4.3$ are also far from obvious, and the provision of a variational principle (3.14) for the steady wave motions that the full Kelvin-Helmholtz model in fact incorporates, even under conditions where the basic state of the model is unstable. This and other aspects of the nonlinear problem in a parametric neighbourhood of $\widehat{\mathrm{C}}=0$, particularly bifurcations of steady waves and their stability, will be treated in Part 2 (Benjamin \& Bridges 1997).

\section{REFERENCES}

Adams, R. A. 1975 Sobolev Spaces. Academic Press.

Aref, H. \& Siggia, E. D. 1980 Vortex dynamics of the two-dimensional turbulent shear layer. $J$. Fluid Mech. 100, 705-737.

Benjamin, T. B. 1972 The stablity of solitary waves. Proc. R. Soc. Lond. A 328, 153-83.

Benjamin, T. B. 1974 Lectures on nonlinear wave motion. Lectures in Applied Mathematics, vol. 15, pp. 3-47. AMS.

BenJamin, T. B. 1984 Impulse, flow force and variational principles. IMA J. Appl. Maths 32, 3-68.

Benjamin, T. B. 1987 Hamiltonian theory for motions of bubbles in an infinite liquid. J. Fluid Mech. 181, 349-379.

Benjamin, T. B. \& BRidges, T. J. 1997 Reappraisal of the Kelvin-Helmholtz problem. Part 2. Interaction of the Kelvin-Helmholtz, superharmonic and Benjamin-Feir instabilities. J. Fluid Mech. 333, 327-373.

Benjamin, T. B. \& Olver, P. J. 1982 Hamiltonian structure, symmetries and conservation laws for water waves. J. Fluid Mech. 125, 137-185.

Bowman, S. 1987 A note on Hamiltonian structure. Math. Proc. Camb. Phil. Soc. 102, 173-189.

Chandrasekhar, S. 1961 Hydrodynamic and Hydromagnetic Stability. Oxford University Press. 
DrazIN, P. G. 1970 Kelvin-Helmholtz instability of finite amplitude. J. Fluid Mech. 42, 321-335.

HaYes, W. D. 1970 Conservation of action and modal wave action. Proc. R. Soc. Lond. A 320, $187-208$.

Higdon, J. J. L. \& Pozrikidis, C. 1985 The self-induced motion of vortex sheets. J. Fluid Mech. 150, 203-231.

Klaassen, G. P. \& Peltier, W. R. 1985 The onset of turbulence in finite-amplitude KelvinHelmholtz billows. J. Fluid Mech. 155, 1-35.

Lamb, H. 1932 Hydrodynamics, 6th edn. Cambridge University Press. (Dover edition 1945.)

Lewis, D., Marsden, J., Montgomery, R. \& Ratiu, T. 1986 The Hamiltonian structure for dynamic free boundary problems. Physica D 18, 391-404.

MeIron, D. \& SafFMAn, P. 1983 Overhanging interfacial gravity waves of large amplitude. J. Fluid Mech. 129, 213-218.

Miles, J. W. 1977 On Hamilton's principle for surface waves. J. Fluid Mech. 83, 153-158.

Peltier, W. R., Hallé, J. \& Clark, T. L. 1978 The evolution of finite-amplitude Kelvin-Helmholtz billows. Geophys. Astrophys. Fluid Dyn. 10, 53-87.

ZAKHAROv, V. E. 1968 Stability of periodic waves of finite amplitude on the surface of a deep fluid. Zh. Prikl. Mekh. Tekh. Fiz. 9, 86-94. (English transl. J. Appl. Math. Tech. Phys. 2, 90.) 